\title{
Enforcing Programming Guidelines with Region Types and Effects ${ }^{\star}$
}

\author{
Serdar Erbatur ${ }^{1}$, Martin Hofmann ${ }^{1}$, and Eugen Zălinescu ${ }^{2}$ \\ 1 Ludwig-Maximilians-Universität, Munich, Bavaria, Germany \\ \{serdar.erbatur, hofmann\}@ifi.lmu.edu \\ 2 Institut für Informatik, Technische Universität München, Germany \\ eugen.zalinescu@in.tum.de
}

\begin{abstract}
We present in this paper a new type and effect system for Java which can be used to ensure adherence to guidelines for secure web programming. The system is based on the region and effect system by Beringer, Grabowski, and Hofmann. It improves upon it by being parametrized over an arbitrary guideline supplied in the form of a finite monoid or automaton and a type annotation or mockup code for external methods. Furthermore, we add a powerful type inference based on precise interprocedural analysis and provide an implementation in the Soot framework which has been tested on a number of benchmarks including large parts of the Stanford SecuriBench.
\end{abstract}

\section{Introduction}

We present in this paper a new type and effect system for Java which can be used to ensure adherence to guidelines for secure web programming such as proper sanitization of externally supplied strings or appropriate authorization prior to access to sensitive data. Unlike its precursors, the system can be freely configured and in this way it can guarantee adherence to a whole host of such guidelines.

The type system is based on the region and effect systems given in 6[13] but improves upon and extends them in a number of ways. First, our system is parametrized by an arbitrary monoid abstracting both string values and sequences of events such as writing certain strings to files or invoking certain framework methods.

Second, in [6] heuristic context information was used in two places: first, in order to index several types for one and the same method and thus to provide a limited amount of polymorphism and secondly in order to determine regions for newly allocated objects. As a side effect this provided for the first time a rigorous type-theoretic underpinning for context-sensitive and points-to analyses. The system presented here keeps heuristic, user-dependent context information for the points-to part, i.e. to determine regions for newly allocated objects, but uses precise and fully automatic interprocedural analysis for method typings.

* This research is funded by the German Research Foundation (DFG) under research grant 250888164 (GuideForce). 
also refine the class $\mathrm{D}$ using two regions into the refined class types D@red and D@green. For each of these we then have refined field and method typings: the $s$ field of D@red objects is typed String@user and so is the parameter of their constructor. The class D@green uses String@ok instead. If there are more fields and methods that we want to differentiate we might need more regions than just two. With these typings in place, we can then see that the program is correct as follows: the variable f1 gets type D@red whereas f2 gets type D@green. Thus, f2.s has type String@ok and the external method call is permitted. We notice that if we had allowed only one region rather than two, i.e. without regions, we would be forced to give field would s the type String@user in view of the assignment to f1. We thus see how the regions provide object sensitivity. If, on the other hand, we had erroneously written putString(f1.s) then, since f1.s has type String@user a type error would have resulted no matter how many regions we use.

Consider now another example in which we need to take into account method effects, type casting, and library methods for which source code is not available.

Example 2. Consider the following method.

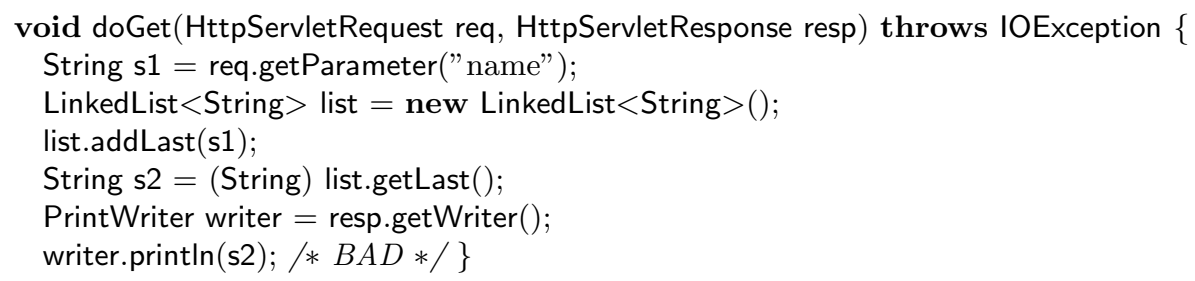

In the last line, a possibly tainted string is written unsanitized.

We use two conceptually different ways to handle external methods whose code is not part of the program being analyzed but comes from some ambient framework or library: (1) builtin methods (like getString) that always take and return strings and are given semantically; (2) external methods (like addLast) that take and return values other than strings and are defined with mockup code.

For the builtin methods we provide their semantic behavior and typing by hand also taking into account tags representing taintedness, the action of sanitization functions, or similar. We also specify event traces produced by them. To this end, we assume an alphabet to represent tags and event traces, and use finite automata and monoids to obtain a finite abstraction of the event traces produced by the program. This eventually allows us to ensure that the set of traces produced by a program will be accepted by a given policy automaton.

Defined external methods are altogether different. We use mockup code to represent their implementation, so as to obtain a model of the part of the ambient framework that is relevant to the analysis. Mockup code is thus analyzed along with the user code. For the given example, we use the following mockup code for linked lists, where we want to impose the abstraction that all their entries are treated the same, e.g., if one entry is tainted then all entries count as tainted. 


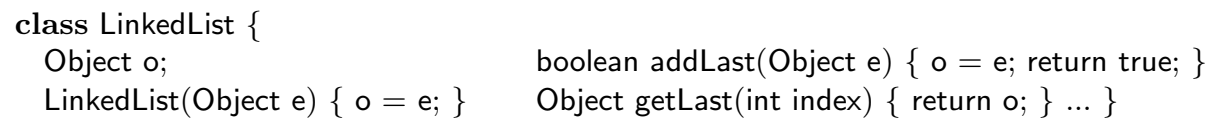

The following mockup code implements the classes HttpServletRequest, etc. and their relevant methods in terms of the builtin methods getString and putString.

class HttpServletRequest \{ String getParameter(String s) \{return getString ()$;\}$ \} class HttpServletResponse $\{$ PrintWriter getWriter ()$\{$ return new PrintWriter ()$;\}\}$ class PrintWriter $\{$ void println(String s) \{return putString(s); \}

An alternative to mockup code which we had also explored consists of ascribing refined types to library methods which then have to be justified manually. However, we found that the expressive power of mockup code is no less than that of arbitrary types and often intuitively clearer. This may be compared to the possibility of approximating stateful behavior of an unknown component with a state machine or alternatively (that would correspond to the use of manually justified types) with a temporal logic formula.

Lastly, to handle casts in general we extended FJEUS in [6] with appropriate semantics and refined type system. Regarding the application scope, we must add that our scope is not limited to guidelines for strings. Currently we are able to formalize guidelines that represent safety properties (see Appendix A for an application to a guideline for authorization) and further plan to extend our approach to liveness and fairness properties.

We stress that our type inference algorithm automatically finds and checks all field and method typings. There is no need for the user to fill in these typings. All a user needs to provide is:

- the policy or guideline to be checked;

- typings or mockup code for external framework methods;

- a context abstraction (usually taken from a few standard ones);

- the set of regions to be used (or their number).

\section{Formalizing Programming Guidelines}

In order to formalize a given guideline to be enforced we select a finite alphabet $\Sigma$ to represent string tags, i.e. annotations that denote some extra information about strings, like taintedness, input kind etc. This same alphabet is also used to represent events generated during the program execution, like writing to a file or the invocation of external methods. One could use different alphabets for tags and events but for simplicity we chose not to do so.

We also assume an infinite set Str of string literals, and a function lit2word : $S t r \rightarrow \Sigma^{*}$ that specifies the tag word $w \in \Sigma^{*}$ for a given string literal.

The operational semantics is then instrumented so that string literals are always paired with their tag words in $\Sigma^{*}$ and evaluation of an expression always results in an event trace also represented as a finite word over $\Sigma$.

Next, we require a finite monoid Mon and a homomorphism [-] : $\Sigma^{*} \rightarrow$ Mon providing a finite abstraction of string tags and event traces. We let Eff := 
$\mathcal{P}($ Mon $)$ and use this abbreviation when referring to event trace abstractions, in contrast to string tag abstractions. We single out a subset Allowed $\subseteq$ Mon consisting of the event traces that are allowed under the guideline to be formalized.

Then, we need to specify a collection of builtin methods such as getString and putString all of which are static and only take string parameters and return a string. We use $f n$ to range over these builtin methods.

For each $n$-ary builtin method $f n$ we assume given a function

$$
\operatorname{sem}(f n):\left(\operatorname{Str} \times \Sigma^{*}\right)^{n} \rightarrow \mathcal{P}\left(\operatorname{Str} \times \Sigma^{*} \times \Sigma^{*}\right) .
$$

The intuition is that when $\left(t, w, w^{\prime}\right) \in \operatorname{sem}(f n)\left(\left(t_{1}, w_{1}\right), \ldots,\left(t_{n}, w_{n}\right)\right)$ then this means that the invocation of the builtin method $f n$ on string arguments $t_{1}, \ldots, t_{n}$ tagged as $w_{1}, \ldots, w_{n}$ can yield result string $t$ tagged as $w$ and leave the event trace $w^{\prime}$. The nondeterminism, i.e. the fact that $\operatorname{sem}(f n)$ returns a set of results allows us for instance to represent user input, incoming requests, or file contents.

Furthermore, we must specify a "typing" in the form of a function

$$
M(f n): M o n^{n} \rightarrow \mathcal{P}(M o n) \times E f f
$$

such that whenever $t_{1}, \ldots, t_{n} \in S t r$ and $w_{1}, \ldots, w_{n} \in \Sigma^{*}$ and $\left(t, w, w^{\prime}\right) \in$ $\operatorname{sem}(f n)\left(\left(t_{1}, w_{1}\right), \ldots,\left(t_{n}, w_{n}\right)\right)$, it holds that $[w] \in U$ and $\left[w^{\prime}\right] \in U^{\prime}$, where $\left(U, U^{\prime}\right)=M(f n)\left(\left[w_{1}\right], \ldots,\left[w_{n}\right]\right)$.

In summary, the formalization of a guideline thus comprises:

- tag and event alphabet, finite monoid 3 allowed subset, and homomorphism,

- semantic functions and typings for builtin methods,

- mockup code for other framework components.

Example 3. Consider the program in Example1. We set $\Sigma=\{$ user, ok $\}$, Mon $=$ $\{\mathrm{T}, \mathrm{U}\}$ ( $\mathrm{T}, \mathrm{U}$ standing for "tainted" / "untainted") with $\mathrm{UU}=\mathrm{U}$ and UT = $\mathrm{TU}=\mathrm{TT}=\mathrm{T}$. Also, [user $]=\mathrm{T},[\mathrm{ok}]=\mathrm{U}$, and Allowed $=\{\mathrm{U}\}$. The semantic function $\operatorname{sem}$ (getString) returns nondeterministically a triple of the form $(t,\langle$ user $\rangle, \varepsilon)$ with $t$ a string, $\varepsilon$ the empty trace, and $\langle-\rangle$ denoting sequences. The semantic function $\operatorname{sem}$ (putString) $(t, w)$ returns the triple $("), \varepsilon,\langle[w]\rangle)$, with empty strings representing void. The typing of getString is $M$ (getString $)()=$ ( $\{[$ user $]\},\{[\varepsilon]\})=(\{\boldsymbol{T}\},\{\mathrm{U}\})$. (Note that $\mathrm{U}$ is the monoid's neutral element.) The typing of putString is $M$ (putString) $(u)=(\{\mathrm{U}\},\{u\})$, for any $u \in$ Mon.

In Appendix $\mathrm{B}$ we give an example of a more elaborate guideline for taintedness analysis, which also takes into account sanitizing functions.

\section{Featherweight Java with Updates, Casts, and Strings}

FJEUCS is a formalized and downsized object-oriented language that captures those aspects of Java that are interesting for our analysis: objects with imperative field updates, and strings. The language is an extension of FJEUS [13] with casts, which itself extends FJEU [15] with strings, which itself extends Featherweight Java [17] with side effects on a heap.

\footnotetext{
${ }^{3}$ Alternatively, the monoid can be generated automatically from the policy automaton (or its complement).
} 


\subsection{Syntax}

The following table summarizes the (infinite) abstract identifier sets in FJEUCS, the meta-variables we use to range over them, and the syntax of expressions. Notice that we only allow variables in various places, e.g., field access so as to simplify the metatheory. By using let-bindings, the effect of nested expressions can be restored (let normal form).

$$
\begin{gathered}
\text { variables: } x, y \in \text { Var } \quad \text { fields: } f \in F l d \quad \text { string literals: str } \in \text { Str } \\
\text { classes: } C, D \in C l s \quad \text { methods: } m \in M t d \quad \text { builtin methods: } f n \in F n \\
\operatorname{Expr} \ni e::=x \mid \text { let } x=e_{1} \text { in } e_{2} \mid \text { if } x_{1}=x_{2} \text { then } e_{1} \text { else } e_{2} \mid \text { null } \mid \text { new } C \\
|(C) e| x . f\left|x_{1} . f:=x_{2}\right| x \cdot m(\bar{y})|f n(\bar{y})| " \text { str" } \mid x_{1}+x_{2}
\end{gathered}
$$

We assume that $\mathrm{Cls}$ contains three distinguished elements, namely Object, String, and NullType 4 In new $C$ expressions, we require that $C \neq$ String and $C \neq$ NullType. Also, in $(C) e$ expressions, $C \neq$ NullType.

An FJEUCS program over the fixed set of builtin methods is defined by the following relations and functions.

$$
\begin{aligned}
\text { subclass relation: } & \prec & \in \mathcal{P} \text { fin }(\mathrm{Cls} \times \mathrm{Cls}) \\
\text { field list: } & \text { fields } & \in C l s \rightarrow \mathcal{P}^{\text {fin }}(\mathrm{Fld}) \\
\text { method list: } & \text { methods } & \in C l s \rightarrow \mathcal{P} \text { fin }(\text { Mtd }) \\
\text { method table: } & \text { mtable } & \in C l s \times M t d \rightarrow \text { Expr } \\
\text { FJEUCS program: } & P & =(\prec, \text { fields, } \text { methods, mtable })
\end{aligned}
$$

FJEUCS is a language with nominal subtyping: $D \prec C$ means $D$ is an immediate subclass of $C$. The relation is well-formed if, when restricted to $C l s \backslash\{$ NullType $\}$ it is a tree successor relation with root Object; thus, multiple inheritance is not allowed. We write $\preceq$ for the reflexive and transitive closure of $\prec$. We also require NullType $\preceq C$ for all $C \in C l s$ and that String is neither a subclass nor a superclass of any proper class (other than NullType, Object, String).

The functions fields and methods describe for each class $C$ which fields and method objects of that class have. The functions are well-formed if for all classes $C$ and $D$ such that $D \preceq C$, fields $(C) \subseteq$ fields $(D)$ and methods $(C) \subseteq$ methods $(D)$, i.e. classes inherit fields and methods from their superclasses. For $C \in\{$ NullType, String $\}$ we require that fields $(C)=$ methods $(C)=\emptyset$. A method table mtable gives for each class and each method identifier its implementation, i.e. the FJEUCS expression that forms the method's body.

A method table is well-formed if the entry $\operatorname{mtable}(C, m)$ is defined for all $m \in$ methods $(C)$. An implementation may be overridden in subclasses for the same number of formal parameters. For simplicity, we do not include overloading. We assume that the formal argument variables of a method $m$ are named $x_{1}^{m}$, $x_{2}^{m}$, etc., besides the implicit and reserved variable this. Only these variables may occur freely in the body of $m$. The number of arguments of $m$ is denoted $\operatorname{ar}(m)$.

\footnotetext{
${ }^{4}$ In Java, the NullType is the type of the expression null, see https://docs.oracle.com/javase/specs/jls/se7/html/jls-4.html\#jls-4.1
} 
A class table $\left(F_{0}, M_{0}\right)$ models FJEUCS standard type system, where types are simply classes. The field typing $F_{0}:(C l s \times F l d) \rightarrow C l s$ assigns to each class $C$ and each field $f \in$ fields $(C)$ the class of the field, which is required to be invariant with respect to subclasses of $C$. The method typing $M_{0}:(C l s \times M t d) \rightarrow C l s{ }^{*} \times C l s$ assigns to each class $C$ and each method $m \in \operatorname{methods}(C)$ a method type, which specifies the classes of the formal argument variables and of the result value.

We make explicit the notion of program point by annotating expressions with expression labels $i \in$ Pos: we write $[e]^{i}$ for FJEUCS expressions, where $e$ is defined as before. An FJEUCS program is well-formed if each expression label $i$ appears at most once in it. In the following, we only consider well-formed programs, and simply write $e$ instead $[e]^{i}$ if the expression label $i$ is not important.

\subsection{Semantics}

A state consists of a store (variable environment or stack) and a heap (memory). Stores map variables to values, while heaps map locations to objects. The only kinds of values in FJEUCS are object locations and null. We distinguish two kinds of objects: ordinary objects contain a class identifier and a valuation of the fields, while string objects are immutable character sequences tagged with a word over the alphabet $\Sigma$. The following table summarizes this state model.

locations: $l \in L o c$ values: $v \in \mathrm{Val}=\operatorname{Loc} \uplus\{$ null $\}$

string objects: $\quad S O b j=S t r \times \Sigma^{*}$ stores: $s \in \mathrm{Var} \rightarrow \mathrm{Val}$

heaps: $h \in L o c \rightarrow O b j \uplus S O b j$

objects: $\quad O b j=C l s \times(F l d \rightarrow V a l)$

The FJEUCS semantics is defined as a big-step relation $(s, h) \vdash e \Downarrow v, h^{\prime} \& w$, which means that, in store $s$ and heap $h$, the expression $e$ evaluates to the value $v$ and modifies the heap to $h^{\prime}$, generating the event trace $w \in \Sigma^{*}$. The operational semantics rules can be found in Appendix D.

\section{Region-based Type and Effect Systems}

\subsection{Refined Types, Effects, and Type System Parameters}

Refined Types We assume a finite set Reg of regions, with Mon $\subseteq$ Reg. We refine the standard object types by annotating objects with sets of regions:

$$
\text { Typ } \ni \tau, \sigma::=C_{R} \quad \text { where } C \in C l s, R \subseteq R e g
$$

such that if $C=$ String then $R \subseteq$ Mon.

A value typed with $C_{R}$, with $R \cap$ Mon $=\emptyset$, intuitively means that it is a location pointing to an ordinary object of class $C$ (or a subclass of $C$ ), and this location is abstracted to a region $r \in R$, but no other region. A value typed with String $_{U}$ (or Object $_{U}$ with $U \subseteq$ Mon) intuitively means that it is a location that refers to a string object that is tagged with a word $w$ such that $[w] \in U$. We use subsets of Reg rather than single elements to account for joining branches of conditionals (including the conditionals implicit in dynamic dispatch). 
Since region sets are an over-approximation of the possible locations where a non-string object resides, and string annotations are an over-approximation of the tags, we define a subtyping relation $<$ : based on set inclusion:

$$
\frac{C \preceq D \quad R \subseteq S}{C_{R}<: D_{S}}
$$

The subtyping relation is extended to type sequences as expected: $\bar{\sigma}<: \bar{\tau}$ iff $|\bar{\sigma}|=|\bar{\tau}|$ and $\sigma_{i}<: \tau_{i}$, for all $i \in\{1, \ldots,|\bar{\sigma}|\}$.

If $R$ is a singleton we call refined types $C_{R}$ atomic (refined) types and denote the set of atomic types by ATyp. We often write $C_{r}$ instead of $C_{\{r\}}$. For a refined type $C_{R}$, let atoms $\left(C_{R}\right):=\left\{C_{r} \mid r \in R\right\}$. For a sequence $\bar{\tau}=\left(\tau_{1}, \ldots \tau_{k}\right)$ of refined types, let atoms $(\bar{\tau}):=\left\{\left(\sigma_{1}, \ldots, \sigma_{k}\right) \mid \sigma_{i} \in \operatorname{atoms}\left(\tau_{i}\right)\right.$, for each $i$ with $\left.1 \leq i \leq k\right\}$.

Type and Effect Lattice We lift the subtyping relation to include effects as well. We define the partial order $\sqsubseteq$ on $\mathcal{L}:=$ Typ $\times \operatorname{Eff}$ by $\left(C_{R}, U\right) \sqsubseteq\left(C_{R^{\prime}}^{\prime}, U^{\prime}\right)$ if and only if $C_{R}<: C_{R^{\prime}}^{\prime}$ and $U \subseteq U^{\prime}$, for any $C, C^{\prime} \in C l s, R, R^{\prime} \subseteq R e g$, and $U, U^{\prime} \subseteq$ Mon. Given two refined types $C_{R}$ and $D_{S}$, we define their join as $C_{R} \sqcup D_{S}=E_{R \cup S}$ where $E$ is the smallest (wrt $\preceq$ ) common superclas:5 of $C$ and $D$. Given two elements $\ell, \ell^{\prime} \in \mathcal{L}$ with $\ell=\left(C_{R}, U\right)$ and $\ell^{\prime}=\left(C_{R^{\prime}}^{\prime}, U^{\prime}\right)$, we define their join, denoted $\ell \sqcup \ell^{\prime}$, by $\left(C_{R} \sqcup C_{R^{\prime}}^{\prime}, U \cup U^{\prime}\right)$. Thus $($ Typ,$\sqcup)$ and $(\mathcal{L}, \sqcup)$ are join-/upper-semilattices. Given $T=\left\{\tau_{1}, \ldots, \tau_{n}\right\} \subseteq$ Typ for some $n \geq 0$, we denote by $\sqcup T$ the type $\tau_{1} \sqcup \tau_{2} \sqcup \cdots \sqcup \tau_{n}$, where by convention $\sqcup T=$ object $_{\text {Reg }}$ when $T=\emptyset$. For $L \subseteq \mathcal{L}$, the notation $\sqcup L$ is defined similarly.

Parameters of the Type and Effect System The following table summarizes the parameters of our system: a set of regions, a set of contexts, a context transfer function, and an object abstraction function. We explain them here briefly.

$$
\begin{array}{rlrl}
\text { Regions (finite): } r, s, t & \in R e g & \\
\text { Contexts (finite): } & z \in C t x & \\
\text { Context transfer function: } & \phi \in C t x \times C l s \times R e g \times M t d \times \text { Pos } \rightarrow \text { Ctx } \\
\text { Object abstraction function: } & \psi \in C t x \times \text { Pos } \rightarrow \text { Reg }
\end{array}
$$

Regions are already defined in Section 4.1. They represent abstract memory locations. Each region stands for zero or more concrete locations. Different regions represent disjoint sets of concrete locations, hence they partition or color the memory. Two pointers to different regions can therefore never alias. Thus the type system serves also as a unifying calculus for pointer analysis.

Let us assume that we have the call graph of a program. A method $m$ is then represented by a node in this graph, and a context corresponds to a possible path that leads to the node. The finite set $C t x$ abstracts these paths. For example, it can be chosen to consists of all locations in the program code or the latter together with, say, the last 3 method calls on the stack (3CFA). The meaning

\footnotetext{
${ }^{5}$ Note that such a class always exists, as $C \preceq$ object, for any $C \in C l s$.
} 
of these contexts is given by the functions $\phi$ and $\psi$ which we explain next. The context transfer function $\phi$ represents the edges in the abstract call graph. It selects a context for the callee based on the caller's context, the class of the receiver object, its region, the method name, and the call site. An object abstraction function $\psi$ assigns a region for a new object, given the allocation site and the current method context. Notice that $C t x$ is an arbitrary finite set and $\phi, \psi$ are arbitrary functions. Their choice does not affect soundness but of course accuracy of the analysis. In [29] a similar factorization of $\phi$ and $\psi$ for callee contexts and object allocation names is presented.

\subsection{Declarative Type System}

As said earlier, the declarative type and effect system is general in the sense that it produces method typings without considering contexts. The method typings along with effects are computed with regard to only method signatures and the associated region information. In addition, new objects are assigned arbitrary regions in a context-insensitive manner.

The typing judgment takes the form $\Gamma \vdash_{\mathrm{d}} \quad e: \tau \& U$, where $e$ is an expression, the variable context (store typing) $\Gamma:$ Var $\rightarrow$ Typ maps variables (at least those in $e$ ) to types, $\tau$ is a type, and $U$ is an element of Eff. The meaning is that, if the values of the variables comply with $\Gamma$ and the evaluation of $e$ terminates successfully, then the result complies with $\tau$, and the event trace generated during this evaluation belongs to one of the equivalence classes in $U$. In particular, if $U \subseteq$ Allowed then $e$ adheres to the guideline. It suffices to perform this latter check for an entry point such as (the body of) a main method.

From a theoretical point of view, the declarative type system forms the basis of our analysis. Once we prove its soundness w.r.t. operational semantics, the soundness of the semi-declarative and algorithmic systems follows directly.

Class Tables A declarative class table $\left(T_{\mathrm{d}}, F, M_{\mathrm{d}}\right)$ fixes a set of $T_{\mathrm{d}} \subseteq$ ATyp of relevant atomic refined types and models FJEUCS's class member types declaratively. The set $T_{\mathrm{d}}$ is required to be closed under "supertyping", that is, for any $C_{r} \in T_{\mathrm{d}}$ and $D \in C l s$ with $C \prec D$, we have that $D_{r} \in T_{\mathrm{d}}$. One can often assume that the set $T_{\mathrm{d}}$ of relevant types contains all types, i.e. $T_{\mathrm{d}}=A T y p$. However, when we take $T_{\mathrm{d}} \subsetneq A T y p$, by having $C \prec D, C_{r} \notin T$, and $D_{r} \in T$, there is an implicit promise that an object with type $D_{r}$ is never an object of type $C_{r}$ that has just been upcast. The usefulness of this feature is illustrated in Appendix H.1.

The field typing $F:($ Fld $\times$ ATyp $) \rightarrow$ Typ assigns to each class $C$, region $r$, and field $f \in$ fields $(C)$ the type $F\left(f, C_{r}\right)$ of the field. The type is required to be invariant with respect to subclasses of $C$. More formally, a field typing $F$ is well-formed if $F\left(f, D_{r}\right)=F\left(f, C_{r}\right)$, for all classes $C$, subclasses $D \preceq C$, regions $r$ with $D_{r} \in T_{\mathrm{d}}$, and fields $f \in$ fields $(C)$. For simplicity, in contrast to [6], we do not use covariant get-types and contravariant set-types for fields.

The declarative method typing $M_{\mathrm{d}}:($ Mtd $\times$ ATyp $) \rightarrow \mathcal{P}\left(\right.$ Typ ${ }^{*} \times$ Typ $\times$ Eff $)$ assigns to each class $C$, region $r$, and method $m \in \operatorname{methods}(C)$, a set $M_{\mathrm{d}}\left(m, C_{r}\right)$ 


$$
\begin{gathered}
\text { TD-NEw } \frac{C_{r} \in T_{\mathrm{d}}}{\Gamma \vdash_{\mathrm{d}} \text { new } C: C_{\{r\}} \&\{[\varepsilon]\}} \\
\text { for all } r \in R \text {, there is }\left(\bar{\sigma}^{\prime}, \tau^{\prime}, U^{\prime}\right) \in M_{\mathrm{d}}\left(m, C_{r}\right) \\
\text { TD-INVOKE } \frac{\text { such that }\left(\bar{\sigma}^{\prime}, \tau^{\prime}, U^{\prime}\right) \sqsubseteq \mathrm{m}(\bar{\sigma}, \tau, U)}{\Gamma, x: C_{R}, \bar{y}: \bar{\sigma} \vdash_{\mathrm{d}} x \cdot m(\bar{y}): \tau \& U} \\
\operatorname{ar}(f n)=n \quad \Gamma\left(x_{1}\right)=\operatorname{String}_{U_{1}}, \ldots, \Gamma\left(x_{n}\right)=\operatorname{String}_{U_{n}} \\
\text { TD-BuiLtin } \frac{M(f n)\left(u_{1}, \ldots, u_{n}\right) \sqsubseteq\left(U, U^{\prime}\right), \text { for all } u_{1} \in U_{1}, \ldots, u_{n} \in U_{n}}{\Gamma \vdash_{\mathrm{d}} f n\left(x_{1}, \ldots, x_{n}\right): \operatorname{String}_{U} \& U^{\prime}}
\end{gathered}
$$

Fig. 1. Selected rules of the declarative type system.

of tuples $(\bar{\sigma}, \tau, U)$, where $\bar{\sigma}$ is a sequence of atomic refined types for the methods' arguments, $\tau$ is the refined type of the result value, and $U$ are the possible effects of the method. Every overriding method should be contravariant in the argument types, covariant in the result class, and have a smaller effect set. Formally, $M_{\mathrm{d}}$ is well-formed if for all classes $C$, subclasses $C^{\prime} \preceq C$, regions $r$ with $C_{r}^{\prime} \in T_{\mathrm{d}}$, and methods $m \in$ methods $(C)$, it holds that

$$
\forall(\bar{\sigma}, \tau, U) \in M_{\mathrm{d}}\left(m, C_{r}\right) . \exists\left(\bar{\sigma}^{\prime}, \tau^{\prime}, U^{\prime}\right) \in M_{\mathrm{d}}\left(m, C_{r}^{\prime}\right) .\left(\bar{\sigma}^{\prime}, \tau^{\prime}, U^{\prime}\right) \sqsubseteq_{\mathrm{m}}(\bar{\sigma}, \tau, U)
$$

where we lift the partial order $\sqsubseteq$ to Typ ${ }^{*} \times$ Typ $\times$ Eff using $\left(\bar{\sigma}^{\prime}, \tau^{\prime}, U^{\prime}\right) \sqsubseteq \mathrm{m}(\bar{\sigma}, \tau, U)$ iff $\bar{\sigma}<: \bar{\sigma}^{\prime}$ and $\left(\tau^{\prime}, U^{\prime}\right) \sqsubseteq(\tau, U)$.

Finally, all types occurring in the field and methods typings are relevant. Formally, $C_{r} \in T_{\mathrm{d}}$, for any $C_{r}$ occurring in the domain of $F$ or $M_{\mathrm{d}}$, and for any $C_{r} \in \operatorname{atoms}\left(C_{R}\right)$ with $C_{R}$ occurring in the image of $F$ or $M_{\mathrm{d}}$.

Type System For space reasons, we only present three of the type rules, given in Figure 1, The complete rules are given in Appendix E.1. In the rule TD-NEW, we choose an arbitrary region as the abstract location of the object allocated by this expression, as long as the respective type is relevant. For method calls, TD-INVOKE requires that for all regions $r \in R$ where the receiver object $x$ may reside, there exists an entry in $M_{\mathrm{d}}$ for the called method and its class such that the resulting type and effect is suitable for the given argument types and the expected result type and effect. In the rule TD-BUiLTin we obtain the refined type of the string returned by a call to the builtin method $f n$, by calling the builtin method typing $M(f n)$ on the tag abstractions of $f n$ 's arguments. Note that also denote by $\sqsubseteq$ the partial order over $\mathcal{P}($ Mon $) \times$ Eff defined by: $\left(R^{\prime}, U^{\prime}\right) \sqsubseteq(R, U)$ iff $R^{\prime} \subseteq R$ and $U^{\prime} \subseteq U$.

An FJEUCS program $P=(\prec$, fields, methods, mtable $)$ is well-typed with respect to the class table $\left(T_{\mathrm{d}}, F, M_{\mathrm{d}}\right)$ if for all classes $C$, regions $r$, methods $m \in$ methods $(C)$, and tuples $(\bar{\sigma}, \tau, U) \in M_{\mathrm{d}}\left(m, C_{r}\right)$, the judgment $\Gamma \vdash_{\mathrm{d}} \operatorname{mtable}(C, m)$ : $\tau \& U$ can be derived with $\Gamma=\left[\right.$ this $\left.\left.\mapsto C_{r}\right] \cup\left[x_{i}^{m} \mapsto \sigma_{i}\right]_{i \in\{1, \ldots, a r}(m)\right\}$. 
Type System Soundness We state next the guarantees provided by the type system, namely that if $\Gamma \vdash_{\mathrm{d}} e: \tau \& U$ can be derived and $U \subseteq$ Allowed, then any event trace of the expression $e$ is allowed by the guideline. See Appendix E.2 for a more general statement of the soundness theorem and its proof.

Theorem 1 (Soundness). Let $P$ be a well-typed program and e an expression with no free variables. If $\Gamma \vdash_{d} e: \tau$ \& $U$ and $(s, h) \vdash e \Downarrow v, h^{\prime} \& w$, for some $\tau, U, v, h^{\prime}$, and $w$, and with $\Gamma$, s, and $h$ the empty mappings, then $[w] \in U$.

\subsection{Semi-declarative Type System}

As already mentioned we rely on heuristic context information in order to infer regions for newly created objects. That is to say, we use the user-provided function $\psi$ in order to select a region for the newly created object based on the position of the statement (expression label) and the current context which is an abstraction of the call stack. Clearly, the use of such arbitrary user-provided decision functions incurs an unavoidable loss of precision. The semi-declarative type system which we now present precisely quantifies this loss of precision. It is still declarative in the sense that types for methods and classes can be arbitrary (sets of simple types), but it is algorithmic in that regions for newly created objects are assigned using the function $\psi$. It also uses the equally user-provided function $\phi$ to manage the context abstractions. The semi-declarative system is therefore sound (Theorem 2) with respect to the declarative one and thus also with respect to the operational semantics via Theorem 1 .

Further down, in Section 4.4 we will then give an algorithmic type system which can be understood as a type inference algorithm presented as a logic program. This algorithmic type system will be shown sound (Theorem 3) and complete (Theorem 4) w.r.t. the semi-declarative system.

Class Tables We define next semi-declarative class tables $\left(T_{\mathbf{s}}, F, M_{\mathbf{s}}\right)$. The set of allowed refined types is parametrized by a context, that is, $T_{\mathrm{s}}$ is a function from Ctx to ATyp. Each set $T_{\mathbf{s}}(z)$ is closed under supertyping as in the declarative case. The field typing $F$ is as in the fully declarative case. The semi-declarative method typing $M_{\mathrm{s}}:(M t d \times C t x \times A T y p) \rightarrow \mathcal{P}\left(\right.$ Typ $^{*} \times$ Typ $\times$ Eff $)$ assigns to each class $C$, region $r$, context $z$, and method $m \in \operatorname{methods}(C)$ a set $M_{\mathbf{s}}\left(m, z, C_{r}\right)$ of tuples $(\bar{\sigma}, \tau, U)$ as in the fully declarative case. As before, overriding methods have to satisfy the following condition: for any context $z$, atomic refined typed $C_{r}^{\prime} \in T_{\mathrm{s}}(z)$, class $C$ with $C^{\prime} \preceq C$, and method $m \in \operatorname{methods}(C)$, it holds that

$$
\forall(\bar{\sigma}, \tau, U) \in M_{\mathbf{s}}\left(m, z, C_{r}\right) . \exists\left(\bar{\sigma}^{\prime}, \tau^{\prime}, U^{\prime}\right) \in M_{\mathbf{s}}\left(m, z, C_{r}^{\prime}\right) .\left(\bar{\sigma}^{\prime}, \tau^{\prime}, U^{\prime}\right) \sqsubseteq \mathrm{m}(\bar{\sigma}, \tau, U) .
$$

Typing Rules The parametric typing judgment takes the form $\Gamma ; z \vdash_{\mathrm{s}} e$ : $\tau \& U$, where $\Gamma, e, \tau$, and $U$ are as for the declarative typing judgment $\Gamma \vdash_{\mathrm{d}}$ $e: \tau \& U$, while $z \in C t x$ is a context. The judgment has the same meaning as before, with the addition that it is relative to the context $z$. The derivation rules 
are the same as for the declarative system (see Appendix E.1. Figure 5), with the addition of the context $z$ in each judgment, except for the two rules given next. In the rule TS-NEw, we choose the region specified by $\psi$ as the abstract location of the object allocated by this expression. For method calls, TS-INVOKE requires that for all regions $r \in R$ where the receiver object $x$ may reside, the method typing in the context selected by $\phi$ is suitable for the given argument types and the expected result type and effect.

$$
\begin{gathered}
\text { TS-New } \frac{r=\psi(z, i) \quad C_{r} \in T_{\mathrm{s}}(z)}{\Gamma ; z \vdash_{\mathrm{s}}[\text { new } C]^{i}: C_{r} \&\{[\varepsilon]\}} \\
\begin{array}{c}
\text { for all } r \in R \text {, there is }\left(\bar{\sigma}^{\prime}, \tau^{\prime}, U^{\prime}\right) \in M_{\mathrm{s}}\left(m, z^{\prime}, C_{r}\right) \\
\text { TS-Invoke } \frac{\text { such that }\left(\bar{\sigma}^{\prime}, \tau^{\prime}, U^{\prime}\right) \sqsubseteq_{\mathrm{m}}(\bar{\sigma}, \tau, U), \text { where } z^{\prime}=\phi(z, C, r, m, i)}{\Gamma, x: C_{R}, \bar{y}: \bar{\sigma} ; z \vdash_{\mathrm{s}}[x . m(\bar{y})]^{i}: \tau \& U}
\end{array}
\end{gathered}
$$

A program $P=(\prec$, fields, methods, mtable $)$ is well-typed w.r.t. the class table $\left(T_{\mathrm{s}}, F, M_{\mathrm{s}}\right)$ if for all classes $C$, contexts $z$, regions $r$, methods $m \in \operatorname{methods}(C)$, and tuples $(\bar{\sigma}, \tau, U) \in M_{\mathrm{s}}\left(m, z, C_{r}\right)$, the judgment $\Gamma ; z \vdash_{\mathrm{s}}$ mtable $(C, m): \tau \& U$ can be derived with $\Gamma=\left[\right.$ this $\left.\mapsto C_{r}\right] \cup\left[x_{i}^{m} \mapsto \sigma_{i}\right]_{i \in\{1, \ldots, \operatorname{ar}(m)\}}$.

Soundness of the semi-declarative type system follows directly from the soundness of declarative type system. That is, since the rules of semi-declarative system are obtained by adding context information to the rules of the declarative system, the soundness result from the previous subsection carries over here.

Theorem 2 (Soundness). If an FJEUCS program is well-typed with respect to a semi-declarative class table $\left(T_{\mathrm{s}}, F, M_{\mathrm{s}}\right)$, then it is well-typed with respect to the corresponding declarative class table $\left(T_{\mathrm{d}}, F, M_{\mathrm{d}}\right)$, where $T_{\mathrm{d}}:=\bigcup_{z \in C t x} T_{\mathrm{s}}(z)$ and $M_{\mathrm{d}}\left(m, C_{r}\right):=\bigcup_{z \in C t x} M_{\mathrm{s}}\left(m, z, C_{r}\right)$.

Regarding the announced lack of completeness with respect to the declarative system, consider e.g. the case where $\psi$ returns one and the same region irrespective of context and position. In this case, two newly created objects:

$$
\begin{aligned}
& \text { StringBuffer } x=\text { new StringBuffer }() ; \\
& \text { StringBuffer } y=\text { new StringBuffer }() ;
\end{aligned}
$$

will be sent to the same region and, e.g., writing an unsanitized string into $x$ followed by outputting y would be overcautiously considered an error. More interesting examples would involve a single allocation statement called several times in different contexts. No matter how fine the abstraction function is chosen, we can always find a situation where two different allocations are sent to the same region because the two contexts are identified by the context abstraction.

\subsection{Algorithmic Type System}

The algorithmic type system is the one we use in our analysis. It returns the most precise typings and is complete with respect to the semi-declarative system. 
Class Tables We defined next algorithmic class tables $\left(T_{\mathrm{a}}, F, M_{\mathrm{a}}\right)$. The sets $T_{\mathrm{a}}(z)$ of relevant refined types per context $z$, and the field typing $F$ are as in the semi-declarative case. The algorithmic method typing $M_{\mathrm{a}}:(M t d \times C t x \times A T y p \times$ $\left.A T y p^{*}\right) \rightarrow T y p \times E f f$ assigns to each class $C$, region $r$, context $z$, and method $m \in$ methods $(C)$, and sequence $\bar{\sigma}$ of atomic refined types for the methods' arguments (i.e. $|\bar{\sigma}|=\operatorname{ar}(m))$, a type and effect value $M_{\mathrm{a}}\left(m, z, C_{r}, \bar{\sigma}\right)$, which specifies the refined type of the result value, as well as the possible effects of the method. Also, as for the other type systems, we require that for any context $z$, atomic refined type $C_{r}^{\prime} \in T_{\mathrm{a}}(z)$, class $C$ with $C^{\prime} \preceq C$, region $r$, and method $m \in$ methods $(C)$, and atomic type sequences $\bar{\sigma}<: \bar{\sigma}^{\prime}$ with $|\bar{\sigma}|=\operatorname{ar}(m)$, it holds that $M_{\mathrm{a}}\left(m, z, C_{r}^{\prime}, \bar{\sigma}^{\prime}\right) \sqsubseteq M_{\mathrm{a}}\left(m, z, C_{r}, \bar{\sigma}\right)$.

Typing Rules The algorithmic typing judgment $\Gamma ; z \vdash_{\mathrm{a}} e: \tau \& U$ takes the same form and has the same meaning as the semi-declarative typing judgment. The rules (see Appendix E.3. Figure 6) are in essence more specialized versions of the ones in the semi-declarative system. For instance, in the rule TA-INVOKE we take the join of all types which are computed with respect to all regions in which the object $x$ may reside and all contexts returned by $\phi$.

$$
\text { TA-Invoke } \frac{(\tau, U)=\bigsqcup\left\{M_{\mathrm{a}}\left(m, z^{\prime}, C_{r}, \bar{\sigma}\right) \mid r \in R, z^{\prime}=\phi(z, C, r, m, i)\right\}}{\Gamma, x: C_{R}, \bar{y}: \bar{\sigma} ; z \vdash_{\mathrm{a}}[x . m(\bar{y})]^{i}: \tau \& U}
$$

An FJEUCS program $P=(\prec$, fields, methods, mtable $)$ is well-typed with respect to the class table $\left(T_{\mathrm{a}}, F, M_{\mathrm{a}}\right)$ if for all classes $C$, contexts $z$, regions $r$, methods $m \in$ methods $(C)$, and sequence $\bar{\sigma}$ of argument types such that $\left(m, z, C_{r}, \bar{\sigma}\right) \in$ $\operatorname{dom}\left(M_{\mathrm{a}}\right)$, the judgment $\Gamma ; z \quad \vdash_{\mathrm{a}} \operatorname{mtable}(C, m): \tau \& U$ can be derived with $\Gamma=\left[\right.$ this $\left.\mapsto C_{r}\right] \cup\left[x_{i}^{m} \mapsto \sigma_{i}\right]_{i \in\{1, \ldots, a r(m)\}}$, where $M_{\mathrm{a}}\left(m, z, C_{r}, \bar{\sigma}\right)=(\tau, U)$.

Theorem 3 (Soundness). If an FJEUCS program $P$ is well-typed with respect to algorithmic class table $\left(T_{\mathrm{a}}, F, M_{\mathrm{a}}\right)$, then it is well-typed with respect to the corresponding semi-declarative class table $\left(T_{\mathrm{a}}, F, M_{\mathrm{s}}\right)$, where

$$
M_{\mathrm{s}}\left(m, z, C_{r}\right):=\left\{(\bar{\sigma}, \tau, U) \mid\left(m, z, C_{r}, \bar{\sigma}\right) \in \operatorname{dom}\left(M_{\mathrm{a}}\right), M_{\mathrm{a}}\left(m, z, C_{r}, \bar{\sigma}\right)=(\tau, U)\right\} .
$$

Soundness of algorithmic type system is inherited from the soundness of semideclarative system. Next, we state the completeness of the algorithmic system with respect to the semi-declarative system

Theorem 4 (Completeness). Let $P$ be a program and $\left(T_{\mathrm{s}}, F, M_{\mathrm{s}}\right)$ a semideclarative class table. If $P$ is well-typed w.r.t. $\left(T_{\mathrm{s}}, F, M_{\mathrm{s}}\right)$ then there is an algorithmic method typing $M_{\mathrm{a}}$ such that the following conditions hold:

- $P$ is well-typed w.r.t. $\left(T_{\mathrm{s}}, F, M_{\mathrm{a}}\right)$,

- " $M_{\mathrm{a}}$ has better types than $M_{\mathrm{s}}$," that is, for each $\left(m, z, C_{r}\right) \in \operatorname{dom}\left(M_{\mathrm{s}}\right)$, each $(\bar{\sigma}, \tau, U) \in M_{\mathrm{s}}\left(m, z, C_{r}\right)$, and each $\bar{\sigma}_{\mathrm{a}} \in \operatorname{atoms}(\bar{\sigma})$, there is a $\left(\tau^{\prime}, U^{\prime}\right) \in$ $M_{\mathrm{a}}\left(m, z, C_{r}, \bar{\sigma}_{\mathrm{a}}\right)$ such that $\left(\tau^{\prime}, U^{\prime}\right) \sqsubseteq(\tau, U)$. 
The desired algorithmic typing $M_{\mathrm{a}}$ is constructed as the least fix-point of the operator that computes the most precise types of method bodies under an assumed method typing. If we had not introduced $\psi$ to resolve the nondeterminism in the rule TD-NEw, we would obtain a typing like $\mathcal{P}($ Typ $\times$ Eff $)$ rather than $($ Typ $\times$ Eff $)$ for the algorithmic types, but then it would not be clear how to compare "best" typings by iteration as we do. For one thing, the cardinality of $\mathcal{P}($ Typ $\times E f f)$ is exponentially larger than that of $($ Typ $\times E f f)$. More importantly there is no obvious ordering on $\mathcal{P}(T y p \times E f f)$ to represent improvement. It seems that the automatic inference of regions without using contexts is a computationally harder problem with a disjunctive flavor requiring for instance SAT-solving but not doable by plain fix-point iteration.

Type Inference Algorithm From the algorithmic type system in Section 4.4, a type inference algorithm can easily be constructed by reading the rules as a functional program. Appendix $\mathrm{F}$ presents a more general type inference algorithm that infers an algorithmic class table for a given program $P$, provided the standard Java types of the program's methods and fields are also given in form of a class table $\left(F_{0}, M_{0}\right)$. As output it returns an algorithmic class table $\left(T_{\mathrm{a}}, F, M_{\mathrm{a}}\right)$ such that $P$ is well-typed with respect to it. Thus the algorithm can be readily used to check whether an expression $e$ follows a guideline.

\section{Experimental Evaluation}

\subsection{Implementation}

We have implemented a variant of the type inference algorithm from Section 4.4 which applies to actual Java programs rather than FJEUCS 6 We describe next the main ingredients of the implementation (see Appendix $G$ for further details). Most importantly, we phrase our analysis as a dataflow problem; it is well-known that type inference can be formulated as a dataflow problem, see e.g. 23. We thus distinguish between intraprocedural analysis and interprocedural analysis.

Our implementation is built on top of the Soot framework 30|20. We benefit from Soot in two ways. First, we use it to transform Java code into Jimple code (a model for Java bytecode), which represents the source language of our analysis. Second, we use Soot's generic intraprocedural dataflow analysis (extending the ForwardFlowAnalysis class) to implement our intraprocedural analysis. The interprocedural analysis is implemented through a standard fix-point iteration using summary tables. Namely, each iteration starts with a summary table, returns a new table, and the two tables are compared; if there is a difference the old one is replaced with the new one and the next iteration starts with the latter. It is clear that the new table extends the old one at each iteration so that a fix-point is reached and hence the analysis terminates 25 .

\footnotetext{
${ }^{6}$ The implementation is available at https://github.com/ezal/TSA
} 
Table 1. Results on the SecuriBench Micro benchmark and on additional examples.

\begin{tabular}{|l|c|l|l|l|c|l|}
\hline test category & $\mathrm{w} / \mathrm{t}$ & comments & & test category & $\mathrm{w} / \mathrm{t}$ & comments \\
\hline \hline Aliasing & $5 / 6$ & $(\mathbf{3})$ & & Inter & $14 / 14$ & \\
\hline Arrays & $8 / 10$ & $(\mathbf{4}):$ matrices & & Pred & $7 / 9$ & $(\mathbf{1})$ \\
\hline Basic & $42 / 42$ & & & Sanitizers & $6 / 6$ & \\
\hline Collections & $14 / 14$ & & & Sessions & $3 / 3$ & \\
\hline DataStructures & $6 / 6$ & & & StrongUpdates & $3 / 5$ & $(\mathbf{2}),($ (4) $)$ synchronize \\
\hline Factories & $3 / 3$ & & & our examples & $24 / 25$ & $(\mathbf{1 1})$ \\
\hline
\end{tabular}

\subsection{Experiments}

We tested and evaluated our tool on the Stanford Securibench Micro benchmark.7 Among the 12 categories of test cases provided by the benchmark, we have analyzed all of them, excluding the one on reflection. Table 1 lists the results obtained. The table also contains a row for the additional examples we considered, which include the ones appearing in this paper. The ' $t$ ' and ' $w$ ' columns denote respectively the number of tests in the category, and how many of those run as expected. Whenever the result is not as expected, the reason is mentioned in the "comments" column, as follows:

(1) Detection of the problem in the test case requires path-sensitivity, while our analysis is path-insensitive.

(2) Field updates are conservatively treated as weak updates, which sometimes leads to false positives, see Appendix $\underline{\mathrm{H} .3}$ for a concrete example.

(3) We believe that this test case was wrongly marked as violating by the benchmark, see Appendix $\mathrm{H} .2$ for details.

(4) We do not yet support two-dimensional arrays and concurrent features.

Each test case is analyzed in at most a few seconds (on a standard computer), except for one case which required 18 seconds. We have also successfully analyzed (in $0.3 \mathrm{sec}$ ) an application from the Stanford Securibench benchmark 8 namely blueblog.

\section{Related Work}

We present a review of recent work in the literature that is relevant to our work. Static analysis has a long history as a research area, which has also been subject to interest from industry. Among many books and surveys available in the literature, we refer to [23] for fundamentals and to [7] for an application-oriented reference. In [7] authors give a detailed explanation of static analysis as part of code review, explain secure programming guidelines and provide exercises with the Fortify code analyzer [3]. Other commercial static analysis tools that help with secure web programming in particular include CheckMarx 1], AppScan 4], and Coverity [2]. These tools check source code against vulnerabilities for various

7 https://suif.stanford.edu/ livshits/work/securibench-micro/

8 https://suif.stanford.edu/ livshits/work/securibench/ 
languages including $\mathrm{C}$ and Java, and provide compliance with guidelines offered by institutions such as OWASP, MISRA, SANS, and Mitre CWE at different levels. Although this fact is expressed in their data sheets, the question of how the commercial tools formalize the guidelines differs from one tool to another and the common practice in general is to hardwire a given guideline into the tool. One of our partially reached goals is to formalize guidelines so that they are specified separately from the source code of our tool and are open to independent review.

Skalka et al. develop a type and effect inference system to enforce tracebased safety properties for higher-order [28] and object-oriented [27/26] languages. They represent event traces of programs as effects and compute a set of constraints which are later fed into off-the-shelf model-checking tools. Thus combining type inference and model checking, Skalka et al. are able to analyze programs with respect to trace properties, in particular flow-sensitive security properties such as access control and resource usage policies. The difference to our approach is that types do not contain the full information about the possible traces but only a finite abstraction which is just fine enough to decide compliance with a given policy. In this way, one may expect more succinct types, more efficient inference, and better interaction with the user. Another difference is that our approach is entirely based on type systems and abstract interpretation and as such does not rely on external model-checking software. This might make it easier to integrate our approach with certification. One can also argue that our approach is more in line with classical type and effect systems where types do not contain programs either but rather succinct abstractions akin to our effects. Our work performs string analysis by reducing the problem to a type inference analysis, wherein to track strings types are extended with regions.

Closer to the present work is the Java(X) system [10]11]. It uses ML-style type inference based on parametrically polymorphic (refined!) types rather than full polymorphism as we do (polymorphic in the sense that a method type is an arbitrary set of simple, refined types). Also the system does not have regionbased tracking of aliasing. On the other hand, Java(X) supports type state [12], i.e. the ability of changing the type of an object or a variable through a modifying action. For this to be sound it is clear that the resource in question must be referenced by a unique pointer and a linear typing discipline is used in loc.cit. to ensure this. We think that type state is essentially orthogonal to our approach and could be added if needed. So far, we felt that for the purpose of enforcing guidelines for secure web programming type state is not so relevant.

\section{Conclusion}

We have developed a type-based analysis to check Java 9 programs against secure programming guidelines. The analysis is sound, albeit incomplete: if the analysis

\footnotetext{
${ }^{9}$ As usual, the formal description of our analysis is in terms of an idealized language, FJEUCS. The implementation takes genuine Java programs. However, it does not support certain features such as concurrency and reflection, which we discuss below.
} 
reports "success", then this means the given program follows the guideline, if it reports "failure" the program may or may not follow the guideline.

Our system is based on the region-based type system in [6] and extends it in several directions. First, we enhanced the refined type system in [6] with an effect mechanism to track event traces, following [13. Second, we parametrized the system over arbitrary guidelines using a) syntactic monoids to abstract traces and string values, b) external static methods given by their semantics, and c) using mockup code to represent library components. Third, we provide a more precise polymorphism for method typings via a precise interprocedural analysis (implemented based on our algorithmic type and effect system) while the system in [6] mainly uses contexts to index refined types and gives only a limited amount of polymorphism requiring user intervention. In [6] contexts provided a novel rigorous type-theoretic foundation for context-sensitive and points-to analyses. We still use contexts to determine regions for newly allocated objects. Fourth, we provide a clear semantic foundation for our analysis and obtain the implementation of type inference in three steps, namely via declarative, semi-declarative, and algorithmic type systems. We establish correctness of our system by proving that each type system is sound with respect to its precursor and that the algorithmic system is also complete w.r.t. the semi-declarative system. Finally, we implemented our system allowing us to analyze actual Java code. We rely on the Soot Framework and analyze on the level of Soot intermediate code (i.e. Jimple) which gives us a variety of language features such as various loop and branching constructs, as well as a limited amount of exception handling for free. While our implementation is for now a prototype, it has allowed us to check a significant part of the SecuriBench Micro benchmark and also a medium-size application.

It is possible to extend our work in several ways. The obvious direction is to formalize more guidelines taken, e.g., from the OWASP or SANS portals to secure web programming. This will further validate our approach to formalization through the described mix of automata, monoids, and modelling of framework code. It will also motivate various extensions to our type-based analysis which we will tackle as needed. We describe here the most important ones.

Reflection. While unconstrained use of reflection would seem to preclude any kind of meaningful static analysis it appears that the use of reflection in actual applications is rather restricted. Being able, for example, to integrate into our analysis the contents of XML-manifests and similar data which is usually processed via reflection would carry a long way.

Path sensitivity. If needed, a limited amount of path sensitivity can be obtained by introducing a class of booleans with subclasses representing true and false. One could then use refined typings for certain predicates which depending on the refined types of their parameters ensure that the result is true or false.

More general automata. Some guidelines may require us to look beyond regular properties. This may require having to use richer specification formalisms, like automata over infinite alphabets [18, to capture authorization per resource rather than per resource kind. Also, we might want to analyze infinitary program properties, e.g. liveness and fairness properties of infinite program traces. 
To this end it should be possible to replace our finite state machines with Büchi automata, and accordingly, our monoids with so-called $\omega$-semigroups. We have successfully carried out initial investigations along those lines [14|16].

Concurrency and higher-order. Other possibilities for extension are concurrency and higher-order functions, i.e. anonymous methods, inner classes, and similar. We believe that ideas from higher-order model checking in type-theoretic form [19] could be fruitful there and initial investigations have confirmed this.

IFDS. Another interesting extension would be to harness the approach to interprocedural analysis IFDS 24] for our type inference which has been integrated with Soot in the form of the Heros plugin. While this would not extend expressivity or accuracy of our approach the increased efficiency might increase its range. As we see it, the gist of IFDS is on the one hand the restriction of function summaries to atoms using distributivity and on the other a clever management of updates to summaries so that repeated recomputation during fixpoint iteration can be reduced to a minimum. Our approach already incorporates the first aspect because types of callees and method parameters are always atomic. We think that this is the reason why our approach works surprisingly well. Nevertheless, we hope that some more efficiency could be squeezed out of the second ingredient and it would be particularly interesting to use Heros as a kind of solver to which we can offload the computation of the final method table.

Certification. While we have not actually fleshed this out, it is clear that the type-theoretic formulation of our analysis lends itself particularly well to independent certification. Indeed, we could write a type checker (which would involve no fixpoint iteration and similar algorithmic techniques at all) for the declarative system and then have the algorithmic type inference generate a derivation in the former system to be checked. This might be much easier than verifying the implementation of the inference engine. Similarly, our soundness proof can be formalized in a theorem prover and declarative typing derivations can then be used to generate formal proofs of correctness; see e.g. the Mobius project 5 .

\section{References}

1. Checkmarx. https://www. checkmarx.com.

2. Coverity. http://www. coverity.com.

3. Fortify. https://software.microfocus.com/en-us/software/sca.

4. IBM AppScan. http://www.ibm.com/software/products/en/appscan-source.

5. G. Barthe, L. Beringer, P. Crégut, B. Grégoire, M. Hofmann, P. Müller, E. Poll, G. Puebla, I. Stark, and E. Vétillard. Mobius: Mobility, ubiquity, security. In $T G C$, volume 4661 of $L N C S$, pages 10-29. Springer, 2007.

6. L. Beringer, R. Grabowski, and M. Hofmann. Verifying pointer and string analyses with region type systems. Computer Languages, Systems \& Structures, 39(2):4965, 2013.

7. B. Chess and J. West. Secure Programming with Static Analysis. Addison-Wesley Professional, first edition, 2007.

8. A. S. Christensen, A. Møller, and M. I. Schwartzbach. Precise analysis of string expressions. In $S A S$, volume 2694 of $L N C S$, pages 1-18. Springer, 2003. 
9. P. Crégut and C. Alvarado. Improving the Security of Downloadable Java Applications With Static Analysis. ENTCS, 141(1):129-144, 2005.

10. M. Degen. JAVA(X) A Type-Based Program Analysis Framework. PhD thesis, Universität Freiburg, June 2011.

11. M. Degen, P. Thiemann, and S. Wehr. Tracking linear and affine resources with Java(X). In ECOOP, volume 4609 of $L N C S$, pages 550-574. Springer, 2007.

12. R. DeLine and M. Fähndrich. Typestates for objects. In ECOOP, volume 3086 of $L N C S$, pages 465-490. Springer, 2004.

13. R. Grabowski, M. Hofmann, and K. Li. Type-based enforcement of secure programming guidelines - code injection prevention at SAP. In FAST, volume 7140 of $L N C S$, pages 182-197. Springer, 2012.

14. M. Hofmann and W. Chen. Abstract interpretation from Büchi automata. In $C S L-L I C S$, pages 51:1-51:10, 2014.

15. M. Hofmann and S. Jost. Type-based amortised heap-space analysis. In ESOP, volume 3924 of $L N C S$, pages 22-37. Springer, 2006.

16. M. Hofmann and J. Ledent. A cartesian-closed category for higher-order model checking. In LICS, pages 1-12, 2017.

17. A. Igarashi, B. C. Pierce, and P. Wadler. Featherweight Java: a minimal core calculus for Java and GJ. ACM Transactions on Programming Languages and Systems, 23(3):396-450, 2001.

18. M. Kaminski and N. Francez. Finite-memory automata. Theoretical Computer Science, 134(2):329-363, 1994.

19. N. Kobayashi and C. L. Ong. A type system equivalent to the modal mu-calculus model checking of higher-order recursion schemes. In LICS, pages 179-188. IEEE Computer Society, 2009.

20. P. Lam, E. Bodden, O. Lhoták, and L. Hendren. The Soot framework for Java program analysis: a retrospective. In CETUS, 2011.

21. T. Lenherr. Taxonomy and Applications of Alias Analysis. Master's thesis, ETH Zürich, 2008.

22. O. Lhoták. Program Analysis using Binary Decision Diagrams. PhD thesis, McGill University, Jan. 2006.

23. F. Nielson, H. R. Nielson, and C. Hankin. Principles of Program Analysis. Springer, 1999.

24. T. W. Reps, S. Horwitz, and S. Sagiv. Precise interprocedural dataflow analysis via graph reachability. In $P O P L$, pages 49-61. ACM Press, 1995.

25. M. Sharir and A. Pnueli. Two approaches to interprocedural data flow analysis. In S. S. Muchnick and N. D. Jones, editors, Program flow analysis - theory and applications, pages 189-233. Prentice-Hall, Englewood Cliffs, NJ, 1981.

26. C. Skalka. Types and trace effects for object orientation. Higher-Order and Symbolic Computation, 21(3):239-282, 2008.

27. C. Skalka, S. F. Smith, and D. V. Horn. A type and effect system for flexible abstract interpretation of Java. ENTCS, 131:111-124, 2005.

28. C. Skalka, S. F. Smith, and D. V. Horn. Types and trace effects of higher order programs. Journal of Functional Programmming, 18(2):179-249, 2008.

29. Y. Smaragdakis, M. Bravenboer, and O. Lhoták. Pick your contexts well: understanding object-sensitivity. In POPL, pages 17-30, 2011.

30. R. Vallée-Rai, P. Co, E. Gagnon, L. J. Hendren, P. Lam, and V. Sundaresan. Soot - a Java bytecode optimization framework. In CASCON. IBM, 1999. 


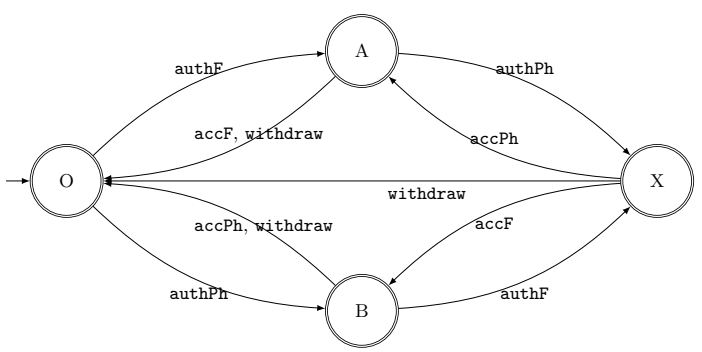

Fig. 2. Authorization policy automaton

\section{A Guideline for Authorization}

Let us look at another guideline which states that any access to sensitive data must only be done after authorization. In this case, the programmer should call authorization methods before access methods.

The following code fragment illustrates the distinction between accesses to different types of resources, namely regular files and phone directories. The guideline is not satisfied.

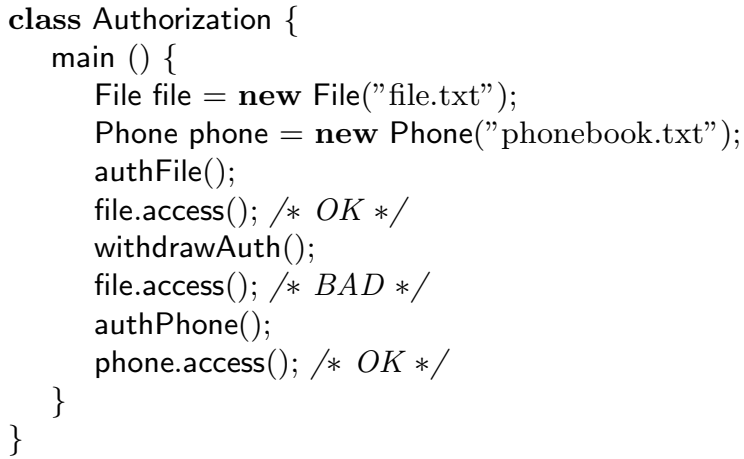

To model this guideline we first consider the possible states which the execution of this program can reach and then the events that trigger state transitions. We define four states; in state 0 no access is allowed, in state A file access is allowed, in state B phone access is allowed and in X both kinds of access are allowed. We assume that successful calls to framework methods given above issue the following events: authF, accF, authPh, accPh, and withdraw. Now we can specify the policy with the finite state machine in Figure 2

Notice that this example is a rather coarse oversimplification. A more realistic version could have different kinds of authorization that may also be withdrawn via system calls. Also, we are not aiming at implementing an access control system, but rather to check that code written with good intentions does not violate guidelines pertaining to authorization. 


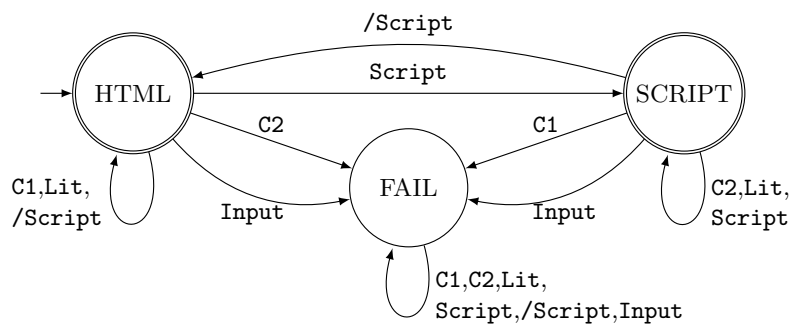

Fig. 3. Policy automaton for taintedness under sanitization

\section{B A More General Guideline for Taintedness}

We reproduce here a guideline first formalized in [13.

In addition to getString and putString from Example1, we also have a function escapeToHtml(input) which escapes '<' and '> ' to '\&lt;' and '\&gt;', and a function escapeToJs(input) escaping user input to be used in a JavaScript context which we don't detail. The tag alphabet is $\Sigma=\{$ Lit, C1, C2, Script,/Script, Input $\}$ with the intention that Input tags unsanitized user input, escapeToHtml results are tagged $\mathrm{C} 1$, escapeToJs results are tagged C2, the tokens " $<$ script $>$ " and $"</$ script $>$ " are tagged eponymously, and everything else is tagged Lit.

The automaton from Figure 3 then specifies the guideline. Intuitively, the automaton is run on the sequence of tags representing how the string fed to putString was obtained and should never go into the failure state.

\section{Additional Details on FJEUCS Syntax}

Consider the program in Example1, The program in FJEUCS and its class table is shown below.

$$
\begin{aligned}
\text { fields }(C) & =\emptyset & & e_{\text {main }}:= \\
\text { fields }(D) & =\{s\} & & \text { let } a=\text { getString }() \text { in } \\
\text { methods }(C) & =\{\text { main }\} & & \text { let } b=\text { test" in } \\
\text { methods }(D) & =\{c D\} & & \text { let } f_{1}=\text { new } D \text { in } \\
\text { mtable }(C, \text { main }) & =e_{\text {main }} & & \text { let }-=f_{1} . c D(a) \text { in } \\
\text { mtable }(D, c D) & =\text { this.s }:=x_{1}^{c D} & & \text { let } f_{2}=\text { new } D \text { in } \\
F_{0}(D, s) & =\text { String } & & \text { let }=f_{2} . c D(b) \text { in } \\
M_{0}(C, \text { main }) & =(\varepsilon, \text { String }) & & \text { putString }\left(f_{2} . s\right) \\
M_{0}(D, c D) & =(\langle\text { String }\rangle, \text { String }) & &
\end{aligned}
$$

In the $e_{\text {main }}$ expression above the underscore denotes some variable not used in the rest of the program. Note that, by convention, methods that normally would not return a value, are assumed to return an empty string. 


$$
\begin{aligned}
& \overline{(s, h) \vdash x \Downarrow s(x), h \& \varepsilon} \quad \overline{(s, h) \vdash \operatorname{null} \Downarrow n u l l, h \& \varepsilon} \\
& \frac{s(x)=s(y) \quad(s, h) \vdash e_{1} \Downarrow v, h^{\prime} \& w}{(s, h) \vdash \text { if } x=y \text { then } e_{1} \text { else } e_{2} \Downarrow v, h^{\prime} \& w} \\
& \frac{s(x) \neq s(y) \quad(s, h) \vdash e_{2} \Downarrow v, h^{\prime} \& w}{(s, h) \vdash \text { if } x=y \text { then } e_{1} \text { else } e_{2} \Downarrow v, h^{\prime} \& w} \\
& \frac{(s, h) \vdash e_{1} \Downarrow v_{1}, h_{1} \& w_{1} \quad\left(s\left[x \mapsto v_{1}\right], h_{1}\right) \vdash e_{2} \Downarrow v_{2}, h_{2} \& w_{2}}{(s, h) \vdash \operatorname{let} x=e_{1} \text { in } e_{2} \Downarrow v_{2}, h_{2} \& w_{1} \cdot w_{2}} \\
& \frac{l \notin \operatorname{dom}(h) \quad F=[f \mapsto n u l l]_{f \in \text { fields }(C)}}{(s, h) \vdash \text { new } C \Downarrow l, h[l \mapsto(C, F)] \& \varepsilon} \\
& \frac{(s, h) \vdash e \Downarrow v, h \& w \quad \text { classOf }_{h}(v) \preceq C}{(s, h) \vdash(C) e \Downarrow v, h \& w} \quad \frac{s(x)=l \quad h(l)=(-, F)}{(s, h) \vdash x . f \Downarrow F(f), h \& \varepsilon} \\
& s(x)=l \quad h(l)=(C, F) \\
& \frac{h^{\prime}=h[l \mapsto(C, F[f \mapsto s(y)])]}{(s, h) \vdash x . f:=y \Downarrow s(y), h^{\prime} \& \varepsilon} \\
& s(x)=l \quad s^{\prime}=[\text { this } \mapsto l] \cup\left[x_{i}^{m} \mapsto s\left(y_{i}\right)\right]_{i \in\{1, \ldots, n\}}
\end{aligned}
$$

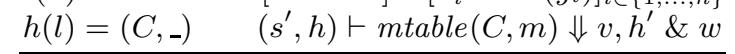

$$
\begin{aligned}
& (s, h) \vdash x . m\left(y_{1}, \ldots, y_{n}\right) \Downarrow v, h^{\prime} \& w \\
& h\left(s\left(y_{i}\right)\right)=\left(s_{t r}, w_{i}\right) \quad l \notin \operatorname{dom}(h) \quad h^{\prime}=h[l \mapsto(s t r, w)] \\
& \operatorname{sem}(f n)\left(\left(\operatorname{str}_{1}, w_{1}\right) \ldots,\left(\operatorname{str}_{n}, w_{n}\right)\right) \ni\left(s t r, w, w^{\prime}\right) \\
& (s, h) \vdash f n\left(y_{1}, \ldots, y_{n}\right) \Downarrow l, h^{\prime} \& w^{\prime}
\end{aligned}
$$

Fig. 4. The FJEUCS operational semantics 


\section{Additional Details on FJEUCS Semantics}

The complete rules for the operational semantics can be found in Figure 4 . A premise involving a partial function, like $s(x)=l$, always implies the side condition $x \in \operatorname{dom}(s)$.

The rules for variables, null, and the conditional are standard. For let expressions, we concatenate the event traces of the two expressions. A new object is allocated at a fresh location with all fields set to null. A cast just checks whether the target type is a subtype of the subject type and raises an exception

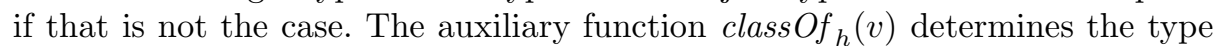
of the subject value $v$ as follows: class $O f_{h}(v)=$ NullType if $v=n u l l$, it is $C$ if $h(v)=\left(C,,_{-}\right) \in O b j$, and it is String if $h(v) \in S O b j$. A field read access returns the field contents, while a field write access updates the heap accordingly (and also evaluates to the written value).

At a method call, a new store is created, consisting of a special variable this and of the method parameters initialized with the values of the passed arguments. The return value, final heap and event trace of the method execution are also the result of the call. A builtin method call allocates a fresh location which stores the result obtained by invoking directly the external semantics of the builtin method on its string arguments.

In the rule for string literals, we rely on lit2word to tag the new string object. The tagging of strings is a homomorphism with respect to string concatenation.

In non-recursive rules, except the one for builtin method calls, we have the empty trace $\varepsilon$ as the generated event trace, as the corresponding expressions do not produce any output.

\section{E Additional Details on the Type Systems}

\section{E.1 Declarative Type and Effect System}

The complete rules for the FJEUCS parametric type system can be found in Figure 5. We briefly explain the rules not presented in the body of the paper. The rule TD-SuB is used to obtain weaker types and effects for an expression. The rule TD-VAR looks up the type of a variable in the context $\Gamma$. In TD-IF, we require that both branches have the same type and effect. This can be obtained in conjunction with the weakening rule TD-SUB. Furthermore, the rule exploits the fact that the two variables must point to the same object (or be null) in the then branch, therefore the intersection of the region sets can be assumed. In TD-LET, we take into account that first the effects of expression $e_{1}$ take place, and then the effects of expression $e_{2}$. The overall effect is thus the concatenation of the sub-effects, where we define $U U^{\prime}=\left\{u \cdot u^{\prime} \mid u \in U, u^{\prime} \in U^{\prime}\right\}$.

When a $C$ object is null, denoted as null, it is given type NullType with an empty region and empty effect via the rule TD-NULL. This is done so that null can be cast to any object (in particular $C$ ) in whatever region, by also using the TD-SUB rule. Note that our type system is not meant to prevent null-pointer 
exceptions, however, being an extension of the standard Java type system, it prevents "method not understood" and similar runtime errors. All soundness statements are conditional on standard termination, i.e. termination without runtime errors 10 The rule TD-CAST allows casting the type of an expression to a subtype or a supertype.

When a field is read (TD-GETF), we look up the type of the field in the $F$ table. As the variable $x$ may point to a number of regions, we need to ensure that $\tau$ is an upper bound of the types of $f$ over all $r \in R$. In contrast, when a field is written (TD-SETF), the written value must have a subtype of the types allowed for that field by the $F$ table with respect to each possible region $r \in R$.

In the rule TD-LiT, we use lit2word to determine the annotation for string literals. In TD-CONCAT, the type of a string obtained by concatenation is defined by concatenating the monoid elements of the types of the concatenated strings.

\section{E.2 Soundness of the Declarative Type System}

We now give a formal interpretation of the typing judgment in form of a soundness theorem. Namely, we prove soundness of declarative type system with respect to operational semantics through interpretation of typing judgment using heap typings. Specifically, our interpretation of the typing judgment $\Gamma \vdash_{\mathrm{d}} e$ : $\tau \& U$ states that whenever a well-typed program is executed on a heap that is well-typed with respect to some heap typing $\Pi$, then the final heap after the execution is well-typed with respect to some possibly larger heap typing $\Pi^{\prime}$.

A heap typing $\Pi:$ Loc $\rightarrow$ ATyp assigns to each heap location a static class (an upper bound of the actual class found at that location) and a region for ordinary objects.

We define a typing judgment for values $\Pi \vdash v: \tau$, which means that according to heap typing $\Pi$, the value $v$ may be typed with $\tau$. In particular, the information in $\Pi(l)$ specifies the type of $l$.

$$
\overline{\Pi \vdash \text { null }: \text { NullType }_{\emptyset}} \quad \frac{\Pi(l)=C_{r}}{\Pi \vdash l: C_{r}} \quad \frac{\Pi \vdash v: \sigma \quad \sigma<: \tau}{\Pi \vdash v: \tau}
$$

Also, the typing judgment of locations is lifted to stores and variable contexts:

$$
\Pi \vdash s: \Gamma \text { iff } \Pi \vdash s(x): \Gamma(x) \text {, for all } x \in \operatorname{dom}(\Gamma)
$$

A heap $h$ is well-typed with respect to a heap typing $\Pi$ and implicitly a declarative class table $\left(T_{\mathrm{d}}, F, M_{\mathrm{d}}\right)$, written $\Pi \vdash h$, if the following conditions hold:

1. at each location, non-string objects are only typed with types in the relevant type set $T_{\mathrm{d}}$ : for any $l \in \operatorname{dom}\left(T_{\mathrm{d}}\right)$, if $\Pi(l)=C_{r}$ and $C \neq$ String, then $C_{\{r\}} \in T_{\mathrm{d}}$,

\footnotetext{
${ }^{10}$ We do not include exceptions in FJEUCS and thus treat any exception as a runtime error. However, the Soot frontend that we use in the implementation (see Section 5.1) compiles away a certain amount of exceptions.
} 
TD-SuB $\frac{\Gamma \vdash_{\mathrm{d}} e: \tau \& U \quad \tau<: \tau^{\prime} \quad U \subseteq U^{\prime}}{\Gamma \vdash_{\mathrm{d}} e: \tau^{\prime} \& U^{\prime}}$

TD-VAR $\overline{\Gamma, x: \tau \vdash_{\mathrm{d}} x: \tau \&\{[\varepsilon]\}}$

TD-IF $\frac{\Gamma, x: C_{R \cap S}, y: D_{R \cap S} \vdash_{\mathrm{d}} e_{1}: \tau \& U \quad \Gamma, x: C_{R}, y: D_{S} \vdash_{\mathrm{d}} e_{2}: \tau \& U}{\Gamma, x: C_{R}, y: D_{S} \vdash_{\mathrm{d}} \text { if } x=y \text { then } e_{1} \text { else } e_{2}: \tau \& U}$

TD-LET $\frac{\Gamma \vdash_{\mathrm{d}} e_{1}: \tau \& U \quad \Gamma, x: \tau \vdash_{\mathrm{d}} e_{2}: \tau^{\prime} \& U^{\prime}}{\Gamma \vdash_{\mathrm{d}} \text { let } x=e_{1} \text { in } e_{2}: \tau^{\prime} \& U U^{\prime}}$

TD-NuLL $\overline{\Gamma \vdash_{\mathrm{d}} \text { null : NullType } \emptyset\{[\varepsilon]\}}$

$$
\begin{gathered}
\operatorname{TD}-\operatorname{New} \frac{C_{r} \in T_{\mathrm{d}}}{\Gamma \vdash_{\mathrm{d}} \text { new } C: C_{\{r\}} \&\{[\varepsilon]\}} \\
\operatorname{TD}-\operatorname{CAST} \frac{\Gamma \vdash_{\mathrm{d}} e: C_{R} \& U \quad C \preceq D \text { or } D \preceq C}{\Gamma \vdash_{\mathrm{d}}(D) e: D_{R} \& U}
\end{gathered}
$$

TD-Invoke $\frac{\text { for all } r \in R, \text { there is }\left(\bar{\sigma}^{\prime}, \tau^{\prime}, U^{\prime}\right) \in M_{\mathrm{d}}\left(m, C_{r}\right) \text { such that }\left(\bar{\sigma}^{\prime}, \tau^{\prime}, U^{\prime}\right) \sqsubseteq_{\mathrm{m}}(\bar{\sigma}, \tau, U)}{\Gamma, x: C_{R}, \bar{y}: \bar{\sigma} \vdash_{\mathrm{d}} x . m(\bar{y}): \tau \& U}$

$\operatorname{ar}(f n)=n \quad \Gamma\left(x_{1}\right)=$ String $_{U_{1}}, \ldots, \Gamma\left(x_{n}\right)=$ String $_{U_{n}}$

TD-Builtin $\frac{M(f n)\left(u_{1}, \ldots, u_{n}\right) \sqsubseteq\left(U, U^{\prime}\right), \text { for all } u_{1} \in U_{1}, \ldots, u_{n} \in U_{n}}{\Gamma \vdash_{\mathrm{d}} f n\left(x_{1}, \ldots, x_{n}\right): \text { String }_{U} \& U^{\prime}}$

TD-GetF $\frac{F\left(f, C_{r}\right)<: \tau, \text { for all } r \in R}{\Gamma, x: C_{R} \vdash_{\mathrm{d}} x . f: \tau \&\{[\varepsilon]\}}$

$\mathrm{TD}-\operatorname{SetF} \frac{\tau<: F\left(f, C_{r}\right), \text { for all } r \in R}{\Gamma, x: C, y: \tau \vdash_{\mathrm{d}} x . f:=y: \tau \&\{[\varepsilon]\}}$

$\operatorname{TD}-\operatorname{LiT} \frac{\operatorname{lit} 2 \text { word }(s t r)=w}{\Gamma \vdash_{\mathrm{d}} " s t r ": \operatorname{String}_{\{[w]\}} \&\{[\varepsilon]\}}$

TD-ConcAT $\frac{\Gamma\left(x_{1}\right)=\text { String }_{U} \quad \Gamma\left(x_{2}\right)=\text { String }_{U^{\prime}}}{\Gamma \vdash_{\mathrm{d}} x_{1}+x_{2}: \text { String }_{U U^{\prime}} \&\{[\varepsilon]\}}$

Fig. 5. The FJEUCS declarative type system 
2. the object at each location is "valid" with respect to the type predicted by $\Pi$ for that location: $l \in \operatorname{dom}(h)$ and $\Pi \vdash h(l): \Pi(l)$, for all $l \in \operatorname{dom}(\Pi)$, where

$$
\begin{aligned}
& \Pi \vdash\left(C, F^{\prime}\right): D_{r} \text { iff } C=D, \operatorname{dom}\left(F^{\prime}\right)=f i e l d s(C), \text { and } \\
& \Pi \vdash F^{\prime}(f): F\left(f, C_{r}\right), \text { for all } f \in f i e l d s(C), \\
& \Pi \vdash(\operatorname{str}, w): \text { String }_{u} \text { iff }[w]=u .
\end{aligned}
$$

Note that the condition $C=D$ is logically equivalent to the requirement that if $\Pi(l)=C_{r}$ then ${\text { class } O f_{h}}_{h}(l)=C$.

The following lemma follows from the well-typedness definition of heaps.

Lemma 1. For any heap $h$, heap typing $\Pi$, value $v$, class $C$, and region set $R$, if $\Pi \vdash h$ and $\Pi \vdash v: C_{R}$, then $\Pi \vdash v: D_{R}$, where $D=\operatorname{class} O f_{h}(v)$.

Proof. The case $v=$ null is trivial, so assume $v \in L o c$. Let $\Pi(v)=E_{r}$ for some class $E$ and region $r$. From $\Pi \vdash v: C_{R}$ we obtain, by rule inversion, that $E_{r}<: C_{R}$. That is, $E \preceq C$ and $r \in R$. From $\Pi \vdash h$, we obtain that $D=E$. Thus $\Pi \vdash v: D_{r}$ by definition and $\Pi \vdash v: D_{R}$ by subsumption.

As the memory locations are determined at runtime, the heap typings cannot be derived statically. Instead, our interpretation of the typing judgment $\Gamma \vdash_{\mathrm{d}}$ $e: \tau \& U$ states that whenever a well-typed program is executed on a heap that is well-typed with respect to some typing $\Pi$, then the final heap after the execution is well-typed with respect to some possibly larger heap typing $\Pi^{\prime}$. The typing $\Pi^{\prime}$ may be larger to account for new objects that may have been allocated during execution, but the type of locations that already existed in $\Pi$ may not change. More formally, a heap typing $\Pi^{\prime}$ extends a heap typing $\Pi$, written $\Pi^{\prime} \sqsupseteq \Pi$, if $\operatorname{dom}(\Pi) \subseteq \operatorname{dom}\left(\Pi^{\prime}\right)$ and $\Pi(l)=\Pi^{\prime}(l)$, for all $l \in \operatorname{dom}(\Pi)$.

Theorem 5 (Soundness Theorem). Let $P$ be a well-typed program. For all $\Pi, \Gamma, \tau, s, h, e, v, h^{\prime}, w$ with

$$
\Gamma \vdash_{d} e: \tau \& U \quad \text { and } \quad \Pi \vdash s: \Gamma \quad \text { and } \quad \Pi \vdash h \quad \text { and } \quad(s, h) \vdash e \Downarrow v, h^{\prime} \& w
$$

there exists some $\Pi^{\prime} \sqsupseteq \Pi$ such that

$$
\Pi^{\prime} \vdash v: \tau \quad \text { and } \quad \Pi^{\prime} \vdash h^{\prime} \quad \text { and } \quad[w] \in U .
$$

Note that Theorem 1 is a corollary of this theorem.

Proof. In 6] a version of this theorem without effects and builtin functions is presented. The result here follows similarly by induction over the sum of the depth of the derivation of the operational semantics judgment and the depth depth of the derivation of the typing judgment.

First, let us consider the case where $\Gamma \vdash_{\mathrm{d}} e: \tau \& U$ has been derived by subtyping rule. By rule inversion we get $\Gamma \vdash_{\mathrm{d}} e: \tau^{\prime} \& U^{\prime}$ where $\tau^{\prime}<: \tau$ and $U^{\prime} \subseteq U$. By induction we obtain $\Pi^{\prime} \vdash v: \tau^{\prime}$ and $\Pi^{\prime} \vdash h^{\prime}$ and $[w] \in U^{\prime}$ for some 
$\Pi^{\prime} \sqsupseteq \Pi$. Since we have $\tau^{\prime}<: \tau$ and $U^{\prime} \subseteq U$, we deduce that $\Pi^{\prime} \vdash v: \tau^{\prime}$ and $[w] \in U^{\prime}$.

Next, we assume that the subtyping rule is not the last used rule of the typing judgment. We perform a case distinction over the last rule used in the derivation of operational semantics judgment. In the rest of the proof, we only consider the cases of object allocations, type casts, and (internal or external) method invocations, the other cases being similar and simpler. We assume that $P$ is well-typed w.r.t. the declarative class table $\left(T_{\mathrm{d}}, F, M_{\mathrm{d}}\right)$.

- $(s, h) \vdash$ new $C \Downarrow l, h[l \mapsto(C, F)] \& \varepsilon$.

The typing judgement is $\Gamma \vdash_{\mathrm{d}}$ new $C: C_{\{r\}} \&\{[\varepsilon]\}$ and thus $C_{\{r\}} \in T_{\mathrm{d}}$. Also, from semantic rule we have $h^{\prime}=h[l \mapsto(C, F)]$ where $l \notin d o m(h)$ and $F=\left[f \mapsto\right.$ null $_{f \in \text { fields }(C)}$. Since $\operatorname{dom}(\Pi) \subseteq \operatorname{dom}\left(h^{\prime}\right)$, we have $l \notin \operatorname{dom}(\Pi)$. We choose $\Pi^{\prime}=\Pi[l \mapsto(C, r)]$, and thus have $\Pi \vdash l: C_{r}$. By definition we

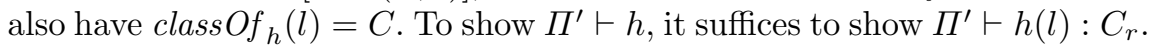
This follows trivially, as $h^{\prime}(l)=(C, F)$ and $C=C$ and $F(f)=$ null for all $f \in \operatorname{dom}(F)$.

$-(s, h) \vdash x . m(\bar{y}) \Downarrow v, h^{\prime} \& w$.

By rule inversion, we know there is a location $l \in \operatorname{dom}(h)$ such that $s(x)=l$ and $h(l)=\left(D,{ }_{-}\right)$, and $\left(s^{\prime}, h\right) \vdash \operatorname{mtable}(D, m) \Downarrow v, h^{\prime} \& w$ where $s^{\prime}=[$ this $\mapsto$ $l] \cup\left[x_{i}^{m} \mapsto s\left(y_{i}\right)\right]_{i \in\{1, \ldots, \operatorname{ar}(m)\}}$.

The typing judgment is $\Gamma, x: C_{R}, \bar{y}: \bar{\sigma} \vdash_{\mathrm{d}} \quad x . m(\bar{y}): \tau \& U$. As $\Pi \vdash s:$ $\left(\Gamma, x: C_{R}, \bar{y}: \bar{\sigma}\right)$, we have $\Pi \vdash l: C_{R}$. By definition of well-typed values w.r.t. $\Pi$, there must exist some class $E$ and some region $r$ with $\Pi(l)=E_{r}$ such that $r \in R$ and $E \preceq C$. We can derive $\Pi \vdash l: C_{\{r\}}$. With $\Pi \vdash h$, we infer that $D=E$ and thus $D \preceq C$. Also, as $\Pi \vdash h$, we have that $E_{r} \in T_{\mathrm{d}}$, that is, $D_{r} \in T_{\mathrm{d}}$.

By rule TD-Invoke, we know there exists a method typing $\left(\bar{\sigma}^{\prime}, \tau^{\prime}, U^{\prime}\right) \in$ $M_{\mathrm{d}}\left(m, C_{r}\right)$ such that $\left(\bar{\sigma}^{\prime}, \tau^{\prime}, U^{\prime}\right) \sqsubseteq_{\mathrm{m}}(\bar{\sigma}, \tau, U)$. As $D \preceq C, D_{r} \in T_{\mathrm{d}}$, and the class table is well-formed, there is a method typing $\left(\bar{\sigma}^{\prime \prime}, \tau^{\prime \prime}, U\right) \in M_{\mathrm{d}}\left(m, D_{r}\right)$ such that $\left(\bar{\sigma}^{\prime \prime}, \tau^{\prime \prime}, U^{\prime \prime}\right) \sqsubseteq_{\mathrm{m}}\left(\bar{\sigma}^{\prime}, \tau^{\prime}, U^{\prime}\right)$. From $\Pi \vdash s:\left(\Gamma, x: C_{R}, \bar{y}: \bar{\sigma}\right)$, it follows $\Pi \vdash s\left(y_{i}\right): \sigma_{i}^{\prime \prime}$ for $i \in\{1, \ldots, \operatorname{ar}(m)\}$.

As $P$ is well-typed, we get $\Gamma^{\prime} \vdash_{\mathrm{d}} \operatorname{mtable}(D, m): \tau^{\prime \prime} \& U$ where $\Gamma^{\prime}=$ $\left[\right.$ this $\left.\mapsto C_{\{r\}}\right] \cup\left[x_{i}^{m} \mapsto \sigma_{i}^{\prime \prime}\right]_{i \in\{1, \ldots, \operatorname{ar}(m)\}}$. From the facts from above, we get $\Pi \vdash s^{\prime}: \Gamma^{\prime}$, so we can finally apply the theorem inductively on the derivation of the semantics and get $[w] \in U$, and $\Pi^{\prime} \vdash v: \tau^{\prime \prime}$ and $\Pi^{\prime} \vdash h^{\prime}$ for some $\Pi^{\prime} \sqsupseteq \Pi$. From $\tau^{\prime \prime}<: \tau$ we obtain $\Pi^{\prime} \vdash v: \tau$.

$-(s, h) \vdash f n(\bar{y}) \Downarrow l, h^{\prime} \& w^{\prime}$, where $f n$ is an external method.

By rule inversion, there is a location $l \notin \operatorname{dom}(h)$, a string literal str $\in$ Str and a words $w \in \Sigma^{*}$ such that $h^{\prime}=h[l \mapsto(s t r, w)]$ and $\left(s t r, w, w^{\prime}\right) \in$ $\operatorname{sem}(f n)\left(\left(\operatorname{str}_{1}, w_{1}\right), \ldots,\left(\operatorname{str}_{n}, w_{n}\right)\right)$, where $n=\operatorname{ar}(f n)$ and $h\left(s\left(y_{i}\right)\right)=\left(\operatorname{str}_{i}, w_{i}\right)$, for each $i$ with $i \in\{1, \ldots, n\}$.

From $\Pi \vdash s: \Gamma$, it follows $\Pi \vdash s\left(y_{i}\right):$ String $_{R_{i}}$. By definition of well-typed values w.r.t. $\Pi$, there must exist some region $r_{i}$ with $\Pi\left(s\left(y_{i}\right)\right)=$ String $_{r_{i}}$ 
and $r_{i} \in R_{i}$. Furthermore, as $\Pi \vdash h$, we obtain that $\left[w_{i}\right]=r_{i}$. Then, from the properties of the typing function $M$ (see Section 2), we get that $[w] \in R$ and $\left[w^{\prime}\right] \in U$, where $(R, U)=M(f n)\left(r_{1}, \ldots, r_{n}\right)$.

We let $\Pi^{\prime}=\Pi[l \mapsto$ (String, $\left.[w])\right]$. Then, as $\Pi \vdash h$, we directly get that $\Pi^{\prime} \vdash h^{\prime}$. Assume that the typing judgment is $\Gamma \vdash_{\mathrm{d}} f n(\bar{y}):$ String $_{R^{\prime}} \& U^{\prime}$ for some $R^{\prime} \subseteq R e g$ and $U^{\prime} \in E f f$. Then, by the rule TD-Builtin, we have $M(f n)\left(r_{1}, \ldots, r_{n}\right) \sqsubseteq\left(R^{\prime}, U^{\prime}\right)$. That is, $(R, U) \sqsubseteq\left(R^{\prime}, U^{\prime}\right)$. Said otherwise $R \subseteq R^{\prime}$ and $U \subseteq U^{\prime}$. It then follows that $[w] \in R^{\prime}$ and $\left[w^{\prime}\right] \in U^{\prime}$. The former conjunct shows that $\Pi^{\prime} \vdash l$ : String $R_{R^{\prime}}$.

$-(s, h) \vdash(C) e \Downarrow v, h \& w$.

The typing judgment is $\Gamma \vdash_{\mathrm{d}}(C) e: C_{R} \& U$. Using rule inversion we get that $\Gamma \vdash_{\mathrm{d}} e: D_{R} \& U$. By the induction hypothesis, there is $\Pi^{\prime} \sqsupseteq \Pi$ such that $\Pi^{\prime} \vdash v: D_{R}, \Pi^{\prime} \vdash h,[w] \in U$. By applying Lemma 1 we get

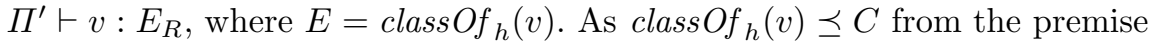
of the operational semantics rule that was used for this case, we obtain $\Pi^{\prime} \vdash v: C_{R}$ by subsumption.

\section{E.3 Algorithmic Type and Effect System}

Figure 6 lists the complete rules of the algorithmic type system. The rules are in essence more specialized versions of the ones in the semi-declarative system. Notice that in the rules TA-IF, TA-Invoke and TA-GetF we compute the least upper bound, i.e. join, of two or more types in premises.

\section{Proof of Completeness}

Proof (of Theorem (4). Given a semi-declarative method typing $M_{\mathrm{s}}$ we define $\Phi\left(M_{\mathrm{s}}\right)$ as the semi-declarative method typing obtained by "best" typing of all the methods according to $M_{\mathrm{s}}$. That is, $\Phi\left(M_{\mathrm{s}}\right)$ returns the most precise type (i.e. the smallest type w.r.t. $\sqsubseteq_{\mathrm{m}}$ in Section 4.3) for each method $m$ in P. Welltypedness w.r.t. $\left(T_{\mathrm{s}}, F, M_{\mathrm{s}}\right)$ essentially says that $\Phi\left(M_{\mathrm{s}}\right) \sqsubseteq M_{\mathrm{s}}$. Accordingly, if $M_{\infty}$ is the least fixpoint of $\Phi$ then $M_{\infty} \sqsubseteq M_{\mathrm{s}}$.

Now, $M_{\infty}$ is obtained as the limit of the chain $\perp:=M_{s_{0}} \sqsubseteq \Phi(\perp):=M_{s_{1}} \sqsubseteq$ $M_{s_{2}} \ldots$ where $M_{s_{(i+1)}}=\Phi\left(M_{s_{i}}\right)$.

Induction on $i$ shows that each typing $M_{s_{i}}$ is actually a function, i.e. for each method $m$, each context $z$, each class $C$, each region $r$, and each $\bar{\sigma}$ there is exactly one region type and effect $(\tau, U)$ such that $(\bar{\sigma}, \tau, U)=M_{s_{i}}\left(m, z, C_{r}\right)$. Now, using the definition of atoms $(\bar{\sigma})$ we can construct $M_{\mathrm{a}}$ as $(\tau, U)=M_{\mathrm{a}}\left(m, z, C_{r}, \bar{\sigma}_{\mathrm{a}}\right)$. Therefore, we can restrict attention to algorithmic method tables throughout.

Regarding well-typedness, from the construction of $M_{\mathrm{a}}$ it follows that if $P$ is well-typed w.r.t. $\left(T_{\mathrm{s}}, F, M_{\mathrm{s}}\right)$ then it is also well-typed w.r.t $\left(T_{\mathrm{s}}, F, M_{\mathrm{a}}\right)$.

\section{F Details of the Type Inference Algorithm}

From the algorithmic type system in Section 4.4 a type inference algorithm can easily be constructed by reading them as a functional program. This algorithm 


$$
\begin{aligned}
& \text { TA-VAR } \overline{\Gamma, x: \tau ; z \vdash_{\mathrm{a}} x: \tau \&\{[\varepsilon]\}} \\
& \Gamma, x: C_{R \cap S}, y: D_{R \cap S} ; z \vdash_{\mathrm{a}} e_{1}: \tau \& U \\
& \text { TA-IF } \frac{\Gamma, x: C_{R}, y: D_{S} ; z \vdash_{\mathrm{a}} e_{2}: \tau^{\prime} \& U^{\prime}}{\Gamma ; z \vdash_{\mathrm{a}} \text { if } x=y \text { then } e_{1} \text { else } e_{2}: \tau \sqcup \tau^{\prime} \& U \cup U^{\prime}} \\
& \text { TA-LET } \frac{\Gamma ; z \vdash_{\mathrm{a}} e_{1}: \tau \& U \quad \Gamma, x: \tau ; z \vdash_{\mathrm{a}} e_{2}: \tau^{\prime} \& U^{\prime}}{\Gamma ; z \vdash_{\mathrm{a}} \text { let } x=e_{1} \text { in } e_{2}: \tau^{\prime} \& U U^{\prime}} \\
& \text { TA-NULL } \frac{}{\Gamma ; z \vdash_{\mathrm{a}} \text { null }: \text { NullType }_{\emptyset} \&\{[\varepsilon]\}} \\
& \text { TA-New } \frac{r=\psi(z, i) \quad C_{r} \in T_{\mathrm{a}}(z)}{\Gamma ; z \vdash_{\mathrm{a}}[\text { new } C]^{i}: C_{\{r\}} \&\{[\varepsilon]\}} \\
& \text { TA-CAST } \frac{\Gamma ; z \vdash_{\mathrm{a}} e: C_{R} \& U \quad C<: D \text { or } D<: C}{\Gamma ; z \vdash_{\mathrm{a}}(D) e: D_{R} \& U} \\
& \text { TA-Invoke } \frac{(\tau, U)=\bigsqcup\left\{M_{\mathrm{a}}\left(m, z^{\prime}, C_{r}, \bar{\sigma}\right) \mid r \in R, z^{\prime}=\phi(z, C, r, m, i)\right\}}{\Gamma, x: C_{R}, \bar{y}: \bar{\sigma} ; z \vdash_{\mathrm{a}}[x \cdot m(\bar{y})]^{i}: \tau \& U} \\
& \operatorname{ar}(f n)=n \quad \Gamma\left(x_{1}\right)=\operatorname{String}_{R_{1}}, \ldots, \Gamma\left(x_{n}\right)=\text { String }_{R_{n}} \\
& \text { TA-Builtin } \frac{(\tau, U)=\bigsqcup\left\{M(f n)\left(r_{1}, \ldots, r_{n}\right) \mid r_{1} \in R_{1}, \ldots, r_{n} \in R_{n}\right\}}{\Gamma ; z \vdash_{\mathrm{a}} f n\left(x_{1}, \ldots, x_{n}\right): \tau \& U} \\
& \text { TA-GETF } \frac{\tau=\bigsqcup\left\{F\left(f, C_{r}\right) \mid r \in R\right\}}{\Gamma, x: C_{R} ; z \vdash_{\mathrm{a}} x . f: \tau \&\{[\varepsilon]\}} \\
& \text { TA-SetF } \frac{\tau<: F\left(f, C_{r}\right), \text { for all } r \in R}{\Gamma, x: C, y: \tau ; z \vdash_{\text {a }} x . f:=y: \tau \&\{[\varepsilon]\}} \\
& \text { TA-LiT } \frac{\text { lit2word }(s t r)=w}{\Gamma ; z \vdash_{\mathrm{a}} " s t r ": \operatorname{String}_{\{[w]\}} \&\{[\varepsilon]\}} \\
& \text { TA-ConcAT } \frac{\Gamma\left(x_{1}\right)=\operatorname{String}_{U} \quad \Gamma\left(x_{2}\right)=\text { String }_{U^{\prime}}}{\Gamma ; z \vdash_{\mathrm{a}} x_{1}+x_{2}: \operatorname{String}_{U U^{\prime}} \&\{[\varepsilon]\}}
\end{aligned}
$$

Fig. 6. The FJEUCS algorithmic type system 
computes the type and effects of any expression $e$, given a typing context $\Gamma$, a method call context $z$, a class table $\left(T_{\mathrm{a}}, F_{\mathrm{a}}, M_{\mathrm{a}}\right)$, and the implicit type systems' parameters. We defer the rather obvious details to Appendix F.1 and henceforth, assume the existence of a procedure typeff, which, when called as typeff $\left(e, \Gamma, z,\left(T_{\mathrm{a}}, F_{\mathrm{a}}, M_{\mathrm{a}}\right)\right)$, returns a tuple $(\tau, U)$.

We present next a more general type inference algorithm, denoted $\mathcal{A}$, that infers an algorithmic class table for a given program $P$, provided the standard Java types of the program's methods and fields are also given in form of a class table $\left(F_{0}, M_{0}\right)$. As output it returns an (algorithmic) class table $\left(T_{\mathrm{a}}, F_{\mathrm{a}}, M_{\mathrm{a}}\right)$ such that $P$ is well-typed with respect to it. Thus the algorithm can be readily used to check whether an expression $e$ follows a guideline. Indeed, it is sufficient to call typeff on the expression $e$ for the newly computed class table and check whether $U \subseteq$ Allowed, where $(\tau, U)$ is the tuple returned by the call to typeff.

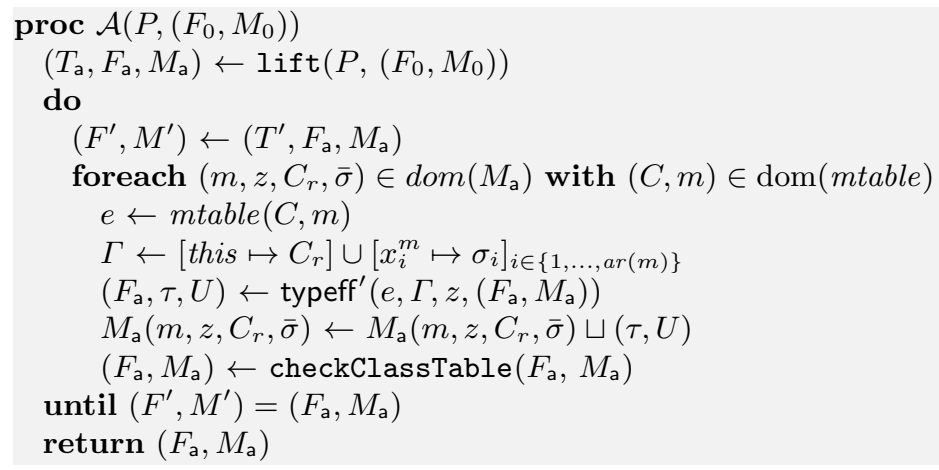

Listing 1.1. The interprocedural analysis.

The pseudo-code of the algorithm $\mathcal{A}$ is given in Listing 1.1. The algorithm computes the field and method typings $\left(F_{\mathrm{a}}, M_{\mathrm{a}}\right)$ iteratively, by a fix-point computation. The tuple $\left(F_{\mathrm{a}}, M_{\mathrm{a}}\right)$ is initialized with the standard class table, by lifting it to an algorithmic class table. More precisely, initially all entries in the tables $F_{\mathrm{a}}$ and $M_{\mathrm{a}}$ are set to the lowest possible elements of $\mathcal{L}$ (w.r.t. $\sqsubseteq$ ). For instance, $F_{\mathrm{a}}\left(f, C_{r}\right)=\left(D_{\emptyset},\{[\varepsilon]\}\right)$, where $D=M_{0}(C, f)$, for any class $C$ and field $f \in$ fields $(C)$. Also, the set $T_{\mathrm{a}}$ of relevant refined types is simply obtained by inspecting all allocations in $P$ in all contexts and adding the corresponding refined types to $T_{\mathrm{a}}$, and then taking the closure of this set by "supertyping".

Next, the fix-point is iteratively computed with a do-until loop. Intuitively, in each iteration, all method bodies of the program are checked against the relevant entries of the method typing $M_{\mathrm{a}}$ and if the check fails than the corresponding entries are updated and their types "weakened". This is performed in the pseudo-code as follows. For each method typing entry, the type and effect of 
the corresponding method body is computed using a variant typeff ${ }^{\prime}$ of the type inference algorithm typeff.

The variant typeff' behaves exactly as typeff, except it also possibly updates the table $F_{\mathrm{a}}$. This may happen for field write sub-expressions $x . f:=y$. Recall that the corresponding type rule requires that $y$ must have a subtype of the types allowed for the field $f$ by the $F_{\text {a }}$ table with respect to each possible region $r \in R$, where $\Gamma(x)=C_{R}$. If this condition is not satisfied then $F_{\mathrm{a}}$ is updated such that it is satisfied, by weakening the offending entry with the type of $y$. Formally, if $\Gamma(y) \nless: F_{\mathrm{a}}\left(f, C_{r}\right)$ for some $r \in R$, then $F_{\mathrm{a}}\left(f, C_{r}\right)$ is set to $F_{\mathrm{a}}\left(f, C_{r}\right) \sqcup \Gamma(y)$.

Once the type and effect $(\tau, U)$ of the analyzed method body is computed, then the current entry of $M_{\mathrm{a}}$ is updated if needed. Finally, the checkClassTable procedure checks whether the tuple $\left(F_{\mathrm{a}}, M_{\mathrm{a}}\right)$ satisfies the constraints from the definition of algorithmic class tables and if not it updates the offending entries so that the constraints are satisfied. For instance, if $C \preceq D$ and $F_{\mathrm{a}}\left(f, C_{r}\right) \neq$ $F_{\mathrm{a}}\left(f, D_{r}\right)$ for some field $f$ and region $r$, then both $F_{\mathrm{a}}\left(f, C_{r}\right)$ and $F_{\mathrm{a}}\left(f, D_{r}\right)$ are set to $F_{\mathrm{a}}\left(f, C_{r}\right) \sqcup F_{\mathrm{a}}\left(f, D_{r}\right)$.

The pseudo-code of the auxiliary procedures lift, checkClassTable, and typeff' can be found later in this section.

Theorem 6. Let $P$ be a program that is well-typed with respect to some class table $\left(F_{0}, M_{0}\right)$. The procedure $\mathcal{A}\left(P,\left(F_{0}, M_{0}\right)\right)$ terminates and returns an algorithmic class table $\left(F_{\mathrm{a}}, M_{\mathrm{a}}\right)$ such that $P$ is well-typed with respect to $\left(F_{\mathrm{a}}, M_{\mathrm{a}}\right)$.

Proof. For termination, we note that in each iteration of the do-until loop at least one of the tables $F_{\mathrm{a}}$ and $M_{\mathrm{a}}$ changes. Furthermore, each change can only "worsen" the type of the changed table entry. Finally, each entry can only be changed a finite number of types, as the last possible change leads to the tuple $\left(\right.$ Object $_{\text {Reg }}, \mathcal{P}($ Mon $\left.)\right)$, which cannot be "worsened".

The correctness of the algorithm relies on the correctness of the typeff' procedure, which is ensured by Theorem 3. Finally, the checkClassTable procedure ensures that the tuple $\left(F_{\mathrm{a}}, M_{\mathrm{a}}\right)$ satisfies the constrains from the definition of algorithmic class tables.

\section{F.1 Pseudocode of the typeff Procedure}

The whole algorithm is presented as imperative style (with pattern matching) pseudo-code in Listings 1.1, 1.3, and 1.2, In the pseudo-code, the symbol := denotes a pattern match followed by an assignment. For instance, if at a point in the pseudo-code $x$ is a fresh variable, while $y$ is already used, then $(x, y):=(a, b)$ first checks that $y$ equals $b$ and then assigns $x$ to $a$. If the check fails, then an exception is raised. The pseudocode of the procedure checkMethodTyping is omitted, being very similar to that of the checkFieldTyping procedure.

Note that in an implementation the tables $M_{\mathrm{a}}$ and $F_{\mathrm{a}}$ would be built onthe-fly. That is, they are not initialized for all possible regions and contexts, but rather an entry is added to the table only when the corresponding value is not the default one. For instance, $F_{\mathrm{a}}\left(f, C_{r}\right)$ only contains entries $\left(E_{R}, U\right)$ with $E \neq D, R \neq \emptyset$, or $U \neq\{[\varepsilon]\}$, where $D=M_{0}(C, f)$. 


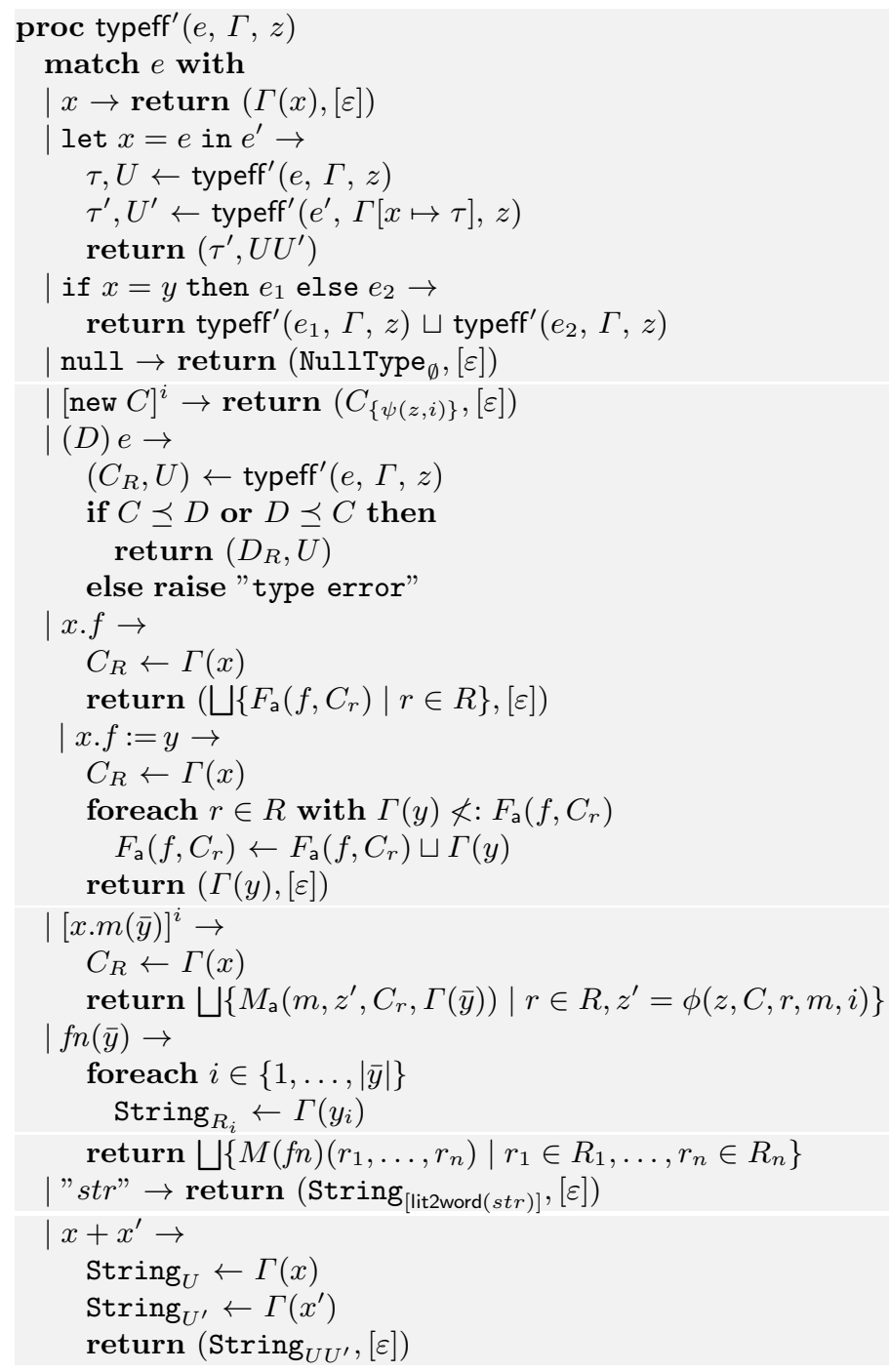

Listing 1.2. The type inference algorithm. 


\section{G Additional Details on the Implementation}

We note that FJEUCS does not feature loops, assignments, and arrays. Instead, loops should be encoded through recursive methods. In the implementation, we use Soot's intraprocedural analysis to handle loops. It is not hard to see that this gives the exact same result as if we would have introduced recursively defined methods for loops. Also, we treat assignments of local variables (but not of fields and array elements) as strong updates, that is, when analyzing an assignment $x:=e$, the type of $x$ becomes $\tau^{\prime}$, where $\tau^{\prime}$ is the type of $e$. Finally, arrays are treated by extending refined types $C_{R}$ to types $C[]_{R}$, with the expected rules: an array access $a[i]$ has type $C_{R}$ if $a$ has type $C_{R}$, and, following an array update $a[i]:=e$, the type of $a$ becomes $D[]_{R^{\prime}}$ with $D_{R^{\prime}}=C_{R} \sqcup \tau^{\prime}$, where $\tau^{\prime}$ is the type of $e$. That is, all array elements have the same refined type. Our mock code for Java collections results in a similar behavior.

The implementation does not support reflection, two-dimensional arrays, concurrent features, and character-level string operations. This means that no guarantees are provided for programs that use the non-supported features. However, our analysis is sound on the fragment we can handle, though not complete. That is, we may obtain false positives, but never obtain false negatives.

The implementation uses the following type system parameters. We let $C t x=$ $\bigcup_{i \in\{0, \ldots, k\}} \operatorname{Pos}^{i}$ and Reg $=C t x \times$ Pos, where $k \geq 0$ is a parameter of the analysis. We take $\psi(z, i)=(z, i)$ and $\phi(z, C, r, m, i)=\left.(i:: z)\right|_{k}$, where $\left.L\right|_{k}$ is the truncation of the list $L$ after the first $k$ elements. Therefore the context of a method call is the call string (i.e. an abstraction of the call stack) restricted to the last $k$ calls; this corresponds the common $k$-CFA abstraction principle. Objects are distinguished their allocation site and by the current context.

\section{H Additional Examples}

\section{H.1 FJ $_{\text {sec }}$ Example}

The following example illustrates that having the set $T_{\mathrm{d}} \subsetneq$ ATyp improve the precision of the analysis. The example is inspired from [26]. There, to treat the corresponding example, "soft subtyping" has been used.

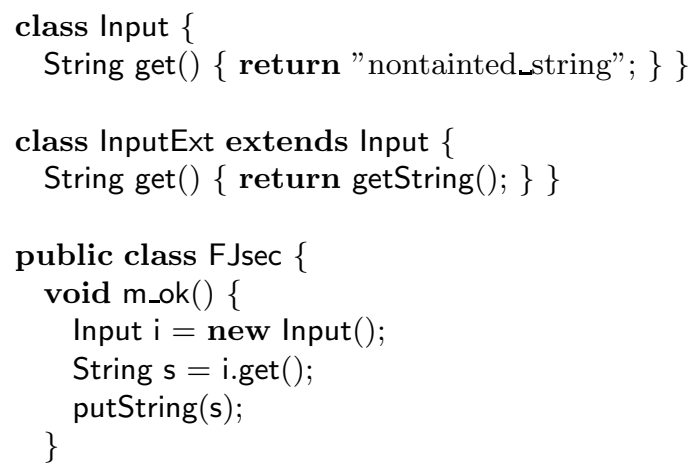




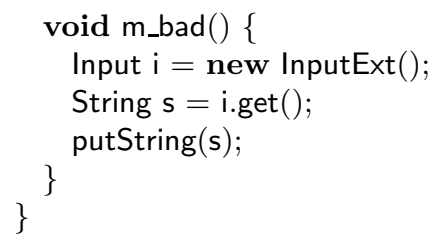

\section{H.2 The SecuriBench test case Aliasing3}

The following code constitutes the Aliasing3 test case from the SecuriBench benchmark, with minor editing for readability.

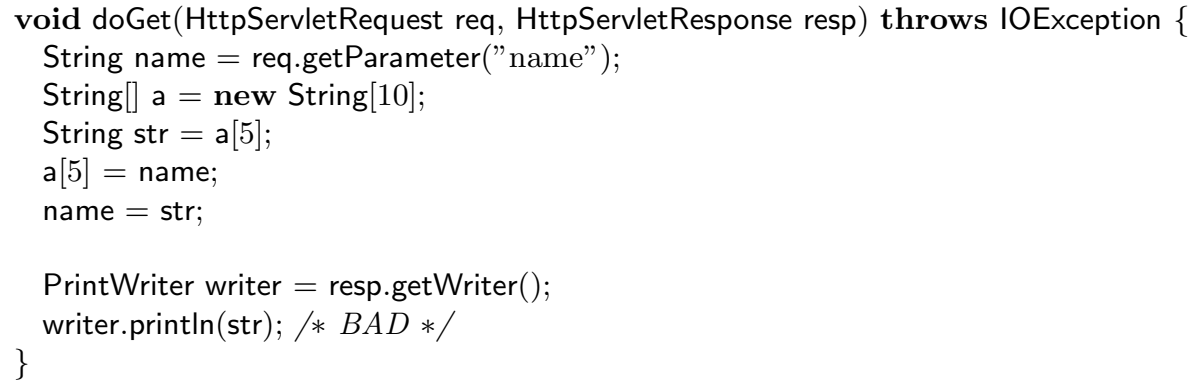

We believe that the test case is wrongly marked as problematic. Indeed, the value of str when given to the print In method is null and this does not constitute a policy violation.

\section{H.3 securibench-micro.Strong_updates3}

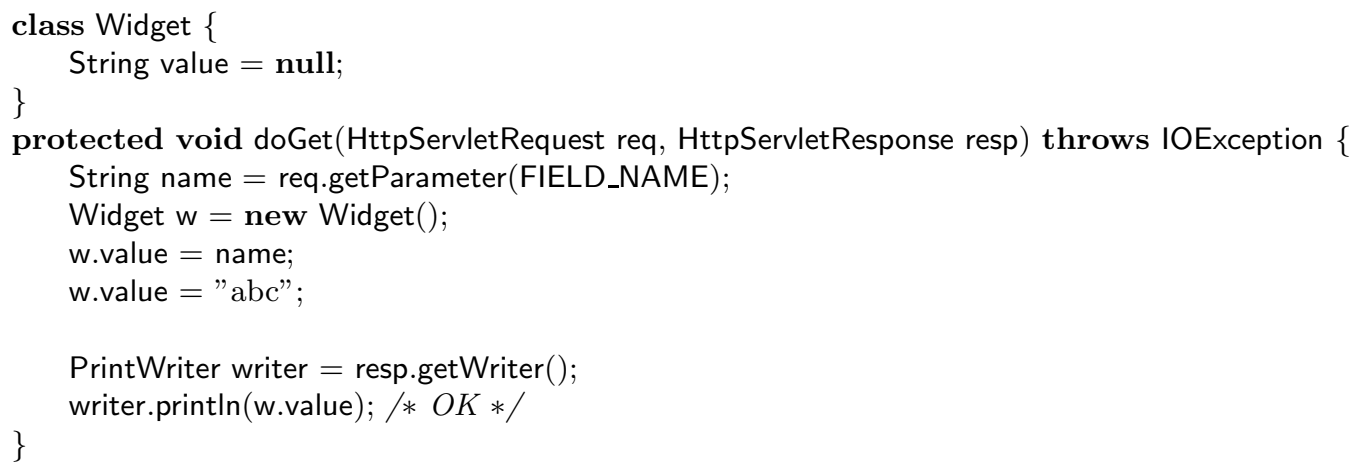

Here the result of our analysis is a false positive (that is, the tool reports a potential violation of the guideline), because field typings are always worsened, never overwritten. 


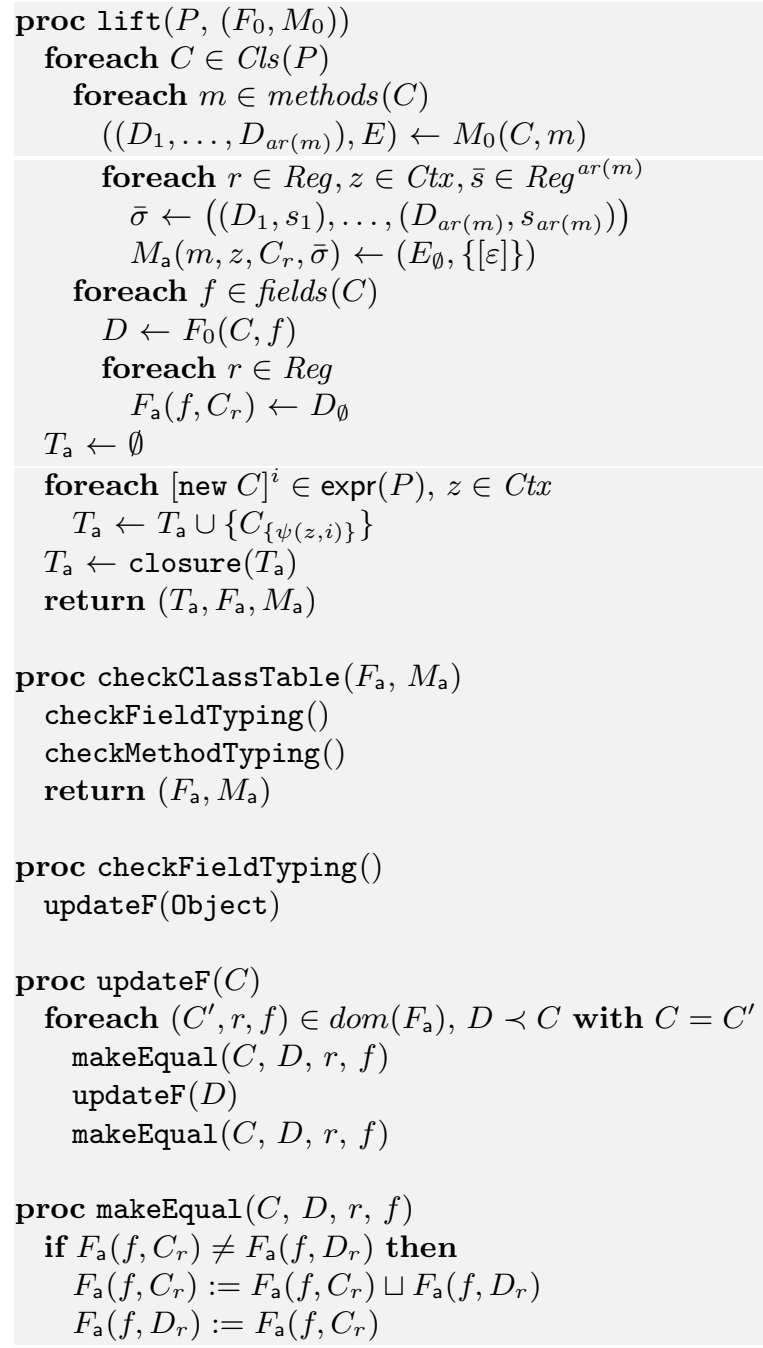

Listing 1.3. Auxiliary procedures for the interprocedural analysis. 